\title{
Equinodermos del Cámbrico de España: situación actual de las investigaciones y perspectivas futuras
}

\author{
Cambrian echinoderms from Spain: current status and future \\ perspectives
}

\author{
S. Zamora ${ }^{1}$
}

RESUMEN

En este trabajo se presenta una revisión histórica de todos los equinodermos cámbricos descubiertos en España, desde su primer hallazgo a finales del siglo XIX y las técnicas actuales que se utilizan para su estudio.

El muestreo sistemático de nuevas localidades cámbricas del Norte de España y la revisión de otras ya conocidas, ha proporcionado más de 2.000 ejemplares de equinodermos. En total se han reconocido taxones pertenecientes a cinco clases: Cincta, Eocrinoidea, Edrioasteroidea, Stylophora y Ctenocystoidea, que muestran la mayor diversidad para este periodo en Gondwana.

Estos hallazgos muestran que los equinodermos alcanzaron su primer pico de diversidad durante el Cámbrico medio. Esto, unido a la amplia distribución paleobiogeográfica de algunos clados, nos advierte de un origen para muchos grupos en el Cámbrico inferior, donde sólo unos pocos taxones han sido descritos a nivel mundial. Por último se dan algunas ideas de hacia dónde se podrían dirigir los esfuerzos en este campo de conocimiento.

Palabras clave: equinodermos, Cámbrico, Gondwana, España, revisión, diversidad

\section{ABSTRACT}

This paper reviews all finds of Cambrian echinoderms from Spain since their first discovery in the XIX century. Recent research techniques used for the study of echinoderms are also described, providing a guide for procedure.

Sampling in new fossiliferous localities from the Cambrian of North Spain and the review of classic ones has yielded more than 2,000 echinoderm fossils. These include species belonging to five major classes, Cincta, Eocrinoidea, Edrioasteroidea, Stylophora and Ctenocystoidea, which show the highest diversity reported from Gondwana.

These results suggest that echinoderms underwent major diversification during the middle Cambrian, with many groups more widely distributed than previously thought. These arguments suggest that many classes originated in the lower Cambrian where only a few taxa have been described. Finally, I offer some suggestions about where research efforts need to be focussed for a better knowledge of Cambrian echinoderm faunas in Gondwana.

Key words: echinoderms, Cambrian, Gondwana, Spain, review, diversity

\section{Introducción}

Los equinodermos son uno de los grupos de invertebrados mejor representado en los ecosistemas marinos actuales y pasados. Este grupo de deu- teróstomos se compone actualmente de cinco clases (Asteroidea, Ofiuroidea, Echinoidea, Holothuroidea y Crinoidea), aunque en el pasado fueron muchas más, y sólo las conocemos a partir de sus fósiles. El registro fósil de los equinodermos se remonta al

\footnotetext{
1 Department of Palaeontology, The Natural History Museum, Cromwell Road, London SW7 5BD, UK. Email: samuel@ unizar.es
} 


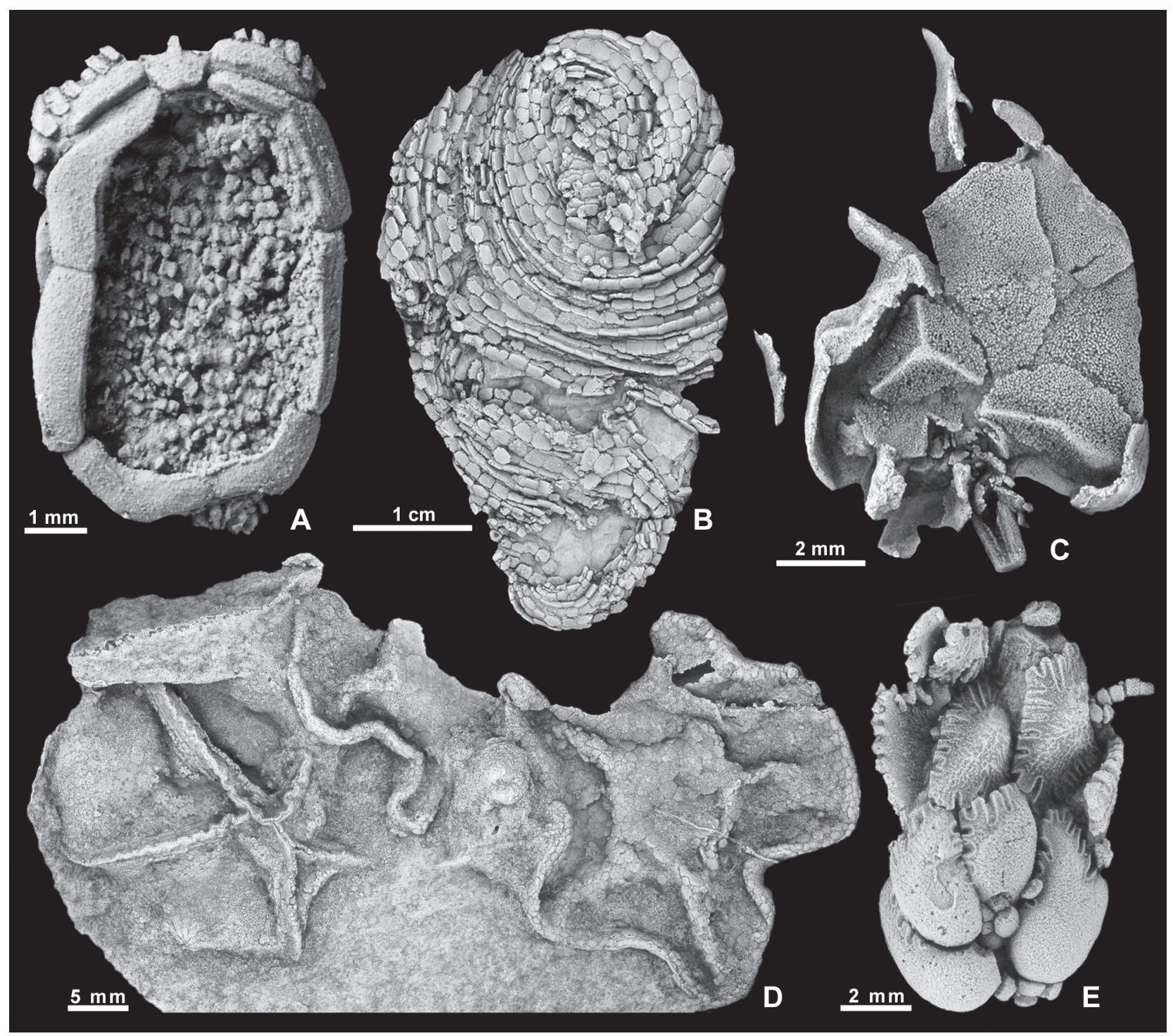

Fig. 1.-Diferentes grupos de equinodermos cámbricos. A. Ctenocistoideo, Ctenocystis utahensis Robison \& Sprinkle del Cámbrico medio de Norteamérica, BMNH E63150. B. Helicoplacoideo, Helicoplacus gilberti Durham \& Caster del Cámbrico inferior de Norteamérica, BMNH E27094. C. Estilóforo, Ceratocystis prosthiakida Rahman, Zamora \& Geyer del Cámbrico medio de Alemania, LfU51733. D. Edrioasteroideos, Stromatocystites pentangularis Pompeckj del Cámbrico medio de Bohemia, BMNH E16004. E. Eocrinoideo, Lichenoides priscus Barrande del Cámbrico medio de Bohemia, BMNH E29346. BMNH: Natural History Museum (London); LfU: Bayerisches Landesamt für Umwelt, München (Alemania).

Cámbrico inferior (530 m.a.) y lo constituyen tres clases: helicoplacoideos, eocrinoideos y edrioasteroideos. Durante el Paleozoico inferior aparecen aproximadamente 30 clados distintos de equinodermos (Sprinkle \& Guensburg, 1997) entre los que se incluyen los primeros representantes de los grupos actuales.

La principal característica de los equinodermos es la presencia de un esqueleto de origen mesodérmico compuesto por numerosas placas de calcita (osículos), que son muy comunes en el registro geológico. La microestructura de estas placas es muy porosa y están formadas por un enrejado de fibras de calcita entrelazadas entre sí, configurando lo que se conoce como estereoma. Los genes responsables de la formación de este esqueleto son únicos y exclusivos de los equinodermos (Bottjer et al., 2006). El sistema vascular acuífero y la simetría radial son otras dos características que se asocian a los equinodermos actuales. 
Los equinodermos son minoritarios en las faunas del Cámbrico. Estos organismos se diversificaron con bastante rapidez, y ya en el Cámbrico medio presentan una diversidad importante, aunque sin llegar a la que alcanzarán durante la gran diversificación del Ordovícico. En un lapso de unos 45 millones de años que supone el Cámbrico inferior y medio, aparecieron siete clases de equinodermos sin que por el momento se conozca su posible ancestro (Sprinkle, 1992). Estas nuevas clases presentan planes corporales muy diferentes, lo que sugiere adaptaciones a modos de vida también muy variados. Generalmente, sus poblaciones están compuestas por numerosos individuos pero con una baja diversidad taxonómica. En muy raras ocasiones, aparece más de un taxón de equinodermos por clase en un mismo nivel, y cuando sucede sus morfologías son muy diferentes (Sprinkle, 1976; Zamora, 2009).

Entre los grupos de equinodermos que tienen su origen en el Cámbrico inferior y medio se encuentran los carpoideos (Clases Soluta, Stylophora, Cincta y Ctenocystoidea), blastozoos (Clase Eocrinoidea), helicoplacoideos (Clase Helicoplacoidea) y edrioasteroideos (Clase Edrioasteroidea) (Fig. 1). Existen aproximadamente 95 especies y 62 géneros de equinodermos formalmente descritos en el Cámbrico de todo el mundo, muchos de los cuales están mal conocidos y/o representados por muy pocos ejemplares.

Este trabajo tiene varios objetivos. Se pretende resumir todas las investigaciones realizadas sobre este grupo en nuestro país, incluyendo muchos datos inéditos. Por otra parte se pretenden explicar las rutinas que se utilizan para el estudio de los equinodermos cámbricos. La presentación de todos los grupos encontrados en el Cámbrico español, servirá para explicar su aportación en cuanto a su diversidad y paleobiogeografía. Tras plantear la problemática actual en el conocimiento de los equinodermos cámbricos gondwánicos, se apuntarán algunos comentarios de hacia dónde se podrían dirigir las investigaciones futuras, y dónde se deberían de focalizar los esfuerzos de los investigadores.

\section{Situación geográfica y geológica}

La Península Ibérica presenta algunos de los mejores y más extensos afloramientos cámbricos de toda Europa (Lotze, 1961; Liñán et al., 2002). De

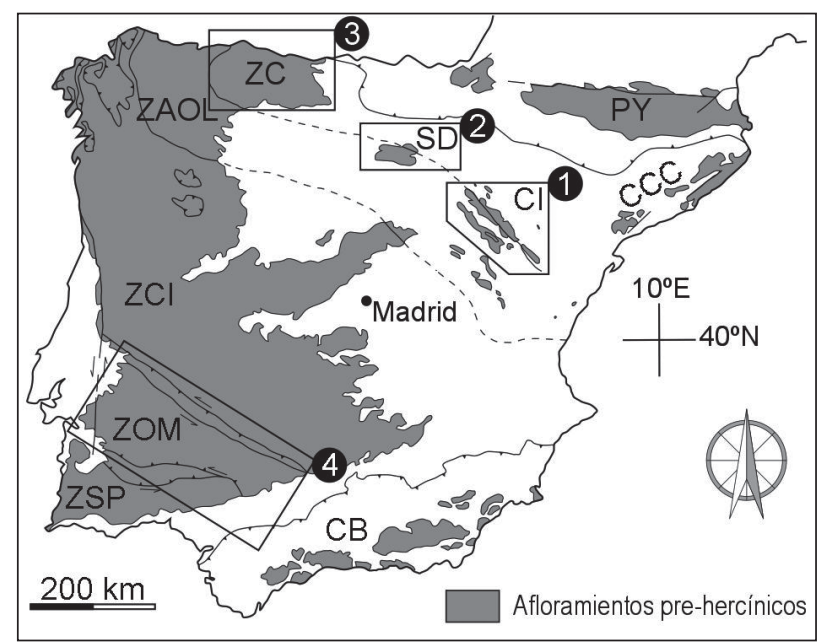

Fig. 2.-Mapa de España donde se muestra la distribución de afloramientos cámbricos, con indicación de las zonas que han proporcionado equinodermos. Modificado de Quesada (1991) y Liñán et al. (2002). 1. Cadenas Ibéricas, 2. Sierra de la Demanda, 3. Zona Cantábrica, 4. Zona de Ossa Morena.

todas las zonas con afloramientos de esta edad, únicamente se tiene constancia de la presencia de restos de equinodermos en las Cadenas Ibéricas, Sierra de la Demanda, Cordillera Cantábrica y Sierra Morena (Fig. 2). Aunque existen algunos equinodermos del Cámbrico inferior, su estudio en profundidad no ha sido abordado todavía, y sólo se comentarán superficialmente.

En las Cadenas Ibéricas se encuentra uno de los registros de equinodermos cámbricos más completos de Europa (Fig. 3). Se han encontrado fósiles en numerosas localidades y formaciones que pueden consultarse en Zamora (2010), aunque en resumen se puede indicar que aparecen restos de equinodermos en las tres formaciones del Grupo Mesones (Valdemiedes, Mansilla y Murero) y en las Formaciones Borobia, Valdeorea y Torcas, del Grupo Acón.

En la Sierra de la Demanda las citas de equinodermos cámbricos son muy escasas, merece la pena comentar que fue de aquí de donde Colchen \& Ubaghs (1969) describieron Oryctoconus lobatus, en la transición Cámbrico-Ordovícico, material que fue más tarde asignado al Ordovícico inferior (Zamora et al. 2009b). Además V. Josopait y U. Schmitz recogieron ejemplares de un cincta del Cámbrico medio que fue clasificado primeramente como Decacystis sp. por el Prof. Ubaghs (en Josopait \& Schmitz, 1971), y más tarde asignado a Gyrocystis platessa por Friedrich (1993). 


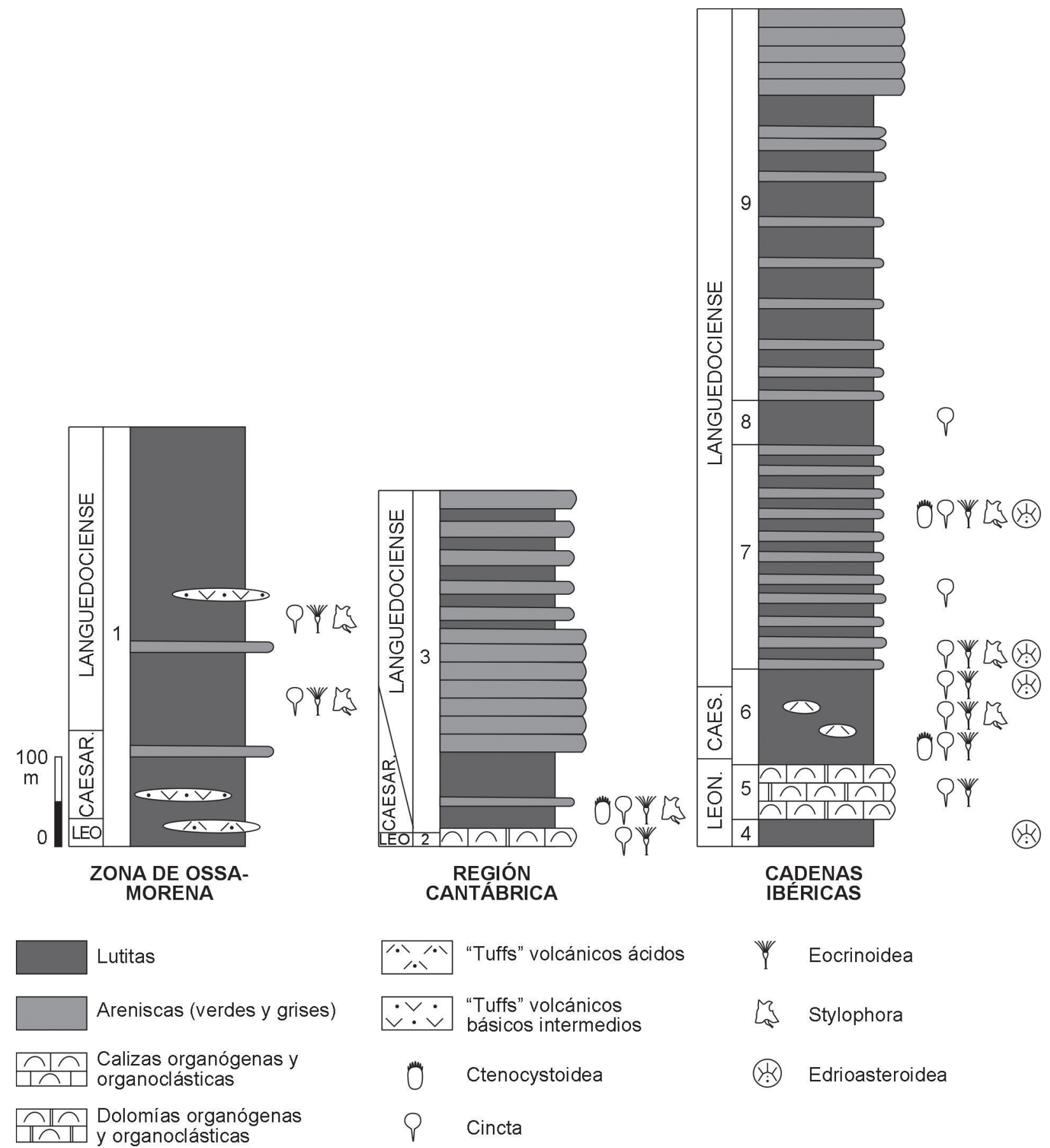

Fig. 3.-Columnas estratigráficas sintéticas de algunas unidades del Cámbrico medio de la Zona Cantábrica, Cadenas Ibéricas y Zona de Ossa Morena, donde se indican los niveles en los que se han recogido restos de equinodermos articulados y el grupo al que pertenecen. Los taxones de cada unidad se detallan en el texto y también pueden consultarse en Zamora (2010). Las columnas están modificadas de Liñán et al. 1997. Números y abreviaciones: 1. «Formación Capas de Playón», 2. Fm. Láncara, 3. Fm. Oville, 4. Fm. Valdemiedes, 5. Fm. Mansilla, 6. Fm. Murero, 7. Fm. Borobia, 8. Fm. Torcas, 9. Fm. Encomienda. Leo. Leoniense, Caesar. Caesaraugustiense. 
En la Cordillera Cantábrica, en concreto en la Zona Cantábrica, los restos de equinodermos cámbricos son muy abundantes en el Miembro Genestosa de la Formación Oville, donde se conoce su presencia desde finales del siglo XIX (Prado et al., 1860). En la infrayacente formación Láncara también se han encontrado equinodermos pero con una menor abundancia de ejemplares completos y un peor estado de conservación (Fig. 3). Para ver un resumen de los taxones reconocidos y las localidades fosilíferas en la Zona Cantábrica puede consultarse Zamora (2010).

En la Zona de Ossa Morena existen dos zonas principalmente fosilíferas desde el punto de vista de los equinodermos: i. la región de Alanís (Sevi1la) donde se encuentra el registro más antiguo de equinodermos cámbricos españoles procedentes del Marianiense, Cámbrico inferior (Ubaghs \& Vizcaïno, 1990); ii. la región de Zafra donde ha sido descrito material procedente del Cámbrico medio de la «Formación Capas de Playón» en la Cubeta de Zafra (Gil Cid \& Domínguez, 1998) (Fig. 3).

\section{Antecedentes históricos}

La primera cita de fósiles de equinodermos en nuestro país es relativamente antigua y se remonta a mediados del siglo XIX cuando Prado et al. (1860) citan la presencia del carpoideo cincta Trochocystites bohemicus?, n. sp. (sic!), en la Cordillera Cantábrica cerca de León, al norte de Sabero y Boñar (Fig. 4). Mallada \& Buitrago (1878) encuentran Trochocystites bohemicus cerca de Belmonte (Oviedo) y en varias localidades leonesas. Mallada (1875) y Monreal (1879) dan un listado con todos los hallazgos donde se había identificado esta especie. Finalmente, Barrois (1882) señala un nuevo yacimiento en Puente Rodical, en el Valle de Narcea (Asturias), lo que constituye la última cita del siglo XIX para estos fósiles en España.

Posteriormente, no será hasta Gislen (1927) cuando de nuevo se estudie este grupo en España, concretamente describe Decacystis hispanica n. gen. n. sp. [actualmente considerado como sinónimo de Gyrocystis platessa de acuerdo con Friedrich (1993)]. El material consistía en un ejemplar y el fragmento de otro procedente del Cámbrico medio de Adrados (León). Hernández-Sampelayo (1935) en su obra «El Sistema Cambriano» fue el primero
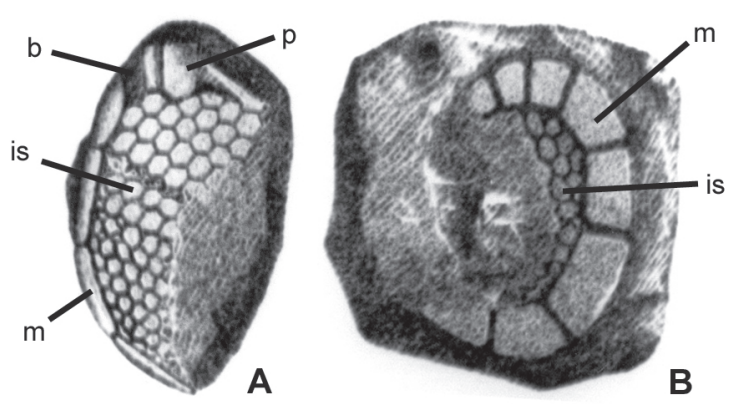

Fig. 4.- -llustración original modificada de Prado et al. 1860, donde se muestran los primeros equinodermos encontrados en el Cámbrico de España, procedentes de la Cordillera Cantábrica. Pertenecen al grupo de los cincta y fueron originariamente identificados como Trochocystites bohemicus ?, n. sp. El ejemplar A representa el molde externo de una cara dorsal, arriba a la izquierda se observa la posición de la boca y en la parte central anterior la impresión del interior del porta. Además se reconoce el interior de las placas infracentrales y algunas marginales. El ejemplar B representa el molde de una cara ventral en la que se reconocen algunas placas infracentrales y varias placas marginales más grandes. Abreviaturas: $b$, boca; $m$, placas marginales; is, placas infracentrales; $p$, porta.

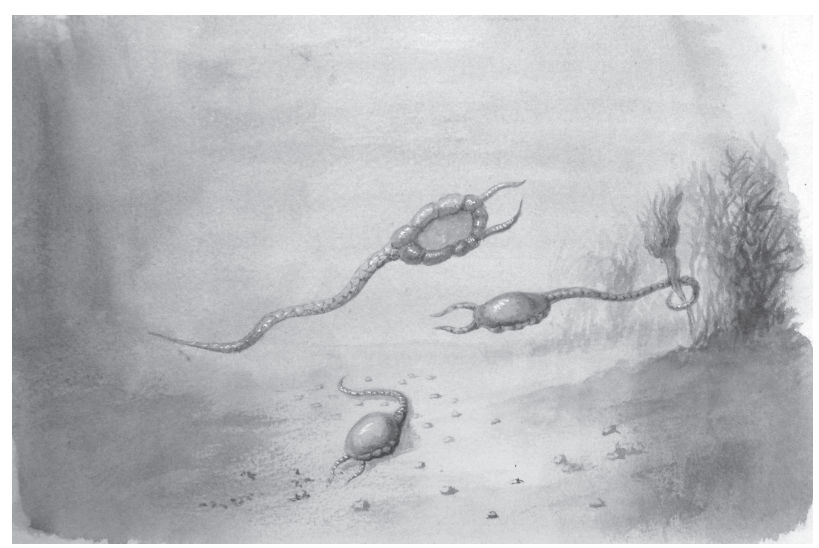

Fig. 5.- Interpretación paleobiológica de los cincta según Meléndez (1952b). En ella aparecen los cincta nadando al ser impulsados con su estela (cola) y con dos apéndices orales. Actualmente se sabe que la estela de los cincta sirve para estabilizarlos en el sustrato y que carecen de apéndices adorales. Imagen Cortesía del Departamento de Paleontología, Universidad Complutense de Madrid.

en citar un cincta, que clasificó como Trochocystites, en la Rambla de Valdemiedes (Zaragoza), junto a él citó otros ejemplares provenientes de Asturias. En este trabajo, el autor determinó erróneamente una librígena del trilobites Paradoxides como si se tratara de una larga estela de un cincta. Ésta identificación errónea llevó a confusión años más tarde a Meléndez (1952b) en sus reconstrucciones sobre los cincta (Fig. 5). 
Un presunto «Trochocystites bohemicus Barrande» fue citado desde antiguo, e incluso confirmado por Regnell (recopilación en Meléndez, 1954), en el supuesto «Cámbrico» del Pirineo catalán. La revisión moderna (Le Menn \& Meléndez, 1988) demostró que el material histórico, junto a otro nuevo, corresponde en su totalidad a placas columnares de crinoideos, muy deformadas tectónicamente, obtenidas en materiales que en realidad pertenecían al Ordovícico Superior.

Es importante destacar que todas las referencias, a excepción de la de Gislen (1927), clasificaban todos los hallazgos de equinodermos cámbricos como pertenecientes al género Trochocystites, el único conocido entonces. La revisión realizada de este material en el trabajo de Friedrich (1993) muestra que muchos de ellos realmente entran dentro de la variabilidad morfológica de la especie Gyrocystis platessa, cuestión que también apuntó Schröeder (1972) en cierta manera. Hasta el momento no se ha encontrado el género Trochocystites fuera de Bohemia, por lo que debe de considerarse endémico de esa región. Meléndez (1952a) traduce la publicación original de Gislen (1927) añadiendo algunos comentarios y en el mismo año Meléndez (1952b) hace una recopilación de todo lo publicado hasta la fecha.

Colchen \& Ubaghs (1969) describen Oryctoconus lobatus, en la transición Cámbrico-Ordovícico de la Sierra de la Demanda. Este trabajo representa la primera cita de pelmatozoos en el Cámbrico de nuestro país. Posteriormente, este género ha sido estudiado en dos trabajos más (Álvaro \& Colchen, 2002; Zamora et al., 2009b), aunque en ellos se reasigna este material al Ordovícico Inferior.

Los trabajos de Schröeder $(1972,1973)$ suponen una puesta al día sobre el conocimiento de los cinctas españoles. En el primero de ellos, se concluye que casi todos los carpoideos conocidos hasta ese momento no pertenecían a Trochocystites bohemicus, sino a Decacystis hispanica. Desde el punto de vista sistemático clasificó un ejemplar figurado por Hernández-Sampelayo (1935, lámina 7, fila central, imagen derecha) y por Meléndez (1952b, lámina 1 fig. 6) como Gyrocystis cf. barrandei n. ssp. También resuelve el problema de la identificación errónea de la larga librígena como una estela de un cincta por Hernández-Sampelayo (1935) y Meléndez (1952b). Por último, aporta los primeros datos sobre la posición estratigráfica de los cincta en España. En el trabajo de 1973, Schröeder (1973) amplia sus aportaciones sobre los cincta en el Cám- brico de España, revisa el material publicado en trabajos previos, junto con el material de las colecciones Josopait y Schmitz depositados en la Universidad de Münster y el material recogido por el propio autor. Entre las novedades, se da a conocer nuevo material de Decacystis hispanica de Ferredal (Asturias) y Láncara de Luna (León), y se describe una nueva especie, Gyrocystis? melendezi en el Cámbrico medio de Valtorres (Ateca, Zaragoza).

Chauvel y Meléndez (en Gutiérrez Marco et al. 1984) figuran un ejemplar de cincta de la zona de Sierra Morena perteneciente a la colección Gil Cid. Este ejemplar fue recogido en las cercanías de Zafra y es de edad Cámbrico medio. El ejemplar representa una cara ventral y fue asignado a Decacystis cf. hispanicus. Este ejemplar fue estudiado años más tarde por Gil Cid \& Domínguez (1995b).

Ubaghs \& Vizcaïno (1990) describen el nuevo subgénero y especie Gogia (Alanisicystis) andalusiae del orden Gogiida; se trata del primer eocrinoideo completo y articulado publicado del Cámbrico español. El material procedía del Marianiense (Cámbrico inferior) de Alanís (Sevilla). El trabajo sigue teniendo total validez.

La monografía de Friedrich (1993) sobre los cincta, es sin duda el trabajo más significativo de todos los que han tratado sobre los equinodermos cámbricos españoles. En esta monografía no sólo se ofrece unas listas de sinonimias adecuadas, en las que se aclaran los problemas nomenclaturales que existían previamente, sino que también se definen varias especies nuevas cuya localidad tipo se encuentra en nuestro país y se incrementa el número de localidades en las que se citan equinodermos. Desde el punto de vista taxonómico, este autor considera que la especie Decacystis hispanica es un sinónimo posterior de Gyrocystis platessa; opina que el ejemplar figurado por Hernández-Sampelayo (1935) y Meléndez (1952b), clasificado por Schröeder (1973) como Gyrocystis cf. barrandei n. ssp. es ahora asignado a Gyrocystis cf. testudiformis n. sp.; la especie Gyrocystis? melendezi Schröeder, 1973 es incluida en el género Sucocystis; se definen tres nuevas especies españolas, incluidas en el género Gyrocystis, cuyas localidades tipo se ubican en las Cadenas Ibéricas: Gyrocystis testudiformis de Jarque (Zaragoza) y, G. badulesiensis y G. erecta, ambos de Badules (Zaragoza). Se define un nuevo género y especie, Progyrocystis disjuncta de Jarque (Zaragoza). Por último, se describe en nomenclatura abierta una nueva forma de Los Barrios de Luna (León), que ha sido estudiada recientemente en base 
a más material (Zamora \& Smith, 2008). Este excelente trabajo taxonómico adolece de un problema importante, ya que en la mayoría de las ocasiones no se especifica la posición estratigráfica concreta de cada taxón.

Sdzuy (1993) ofrece importantes datos, tanto sistemáticos como bioestratigráficos, acerca de los equinodermos cincta españoles, junto con las evidencias más antiguas de este grupo a nivel mundial. En este trabajo se describen dos nuevos géneros y tres nuevas especies procedentes del Cámbrico medio de la presa de Porma y Soto de Caso (León y Asturias): Sotocinctus ubagshi, Asturicystis jaekeli y Trochocystoides planus. De estas tres especies, sólo de Asturicystis se presenta material suficiente, mientras que de Sotocinctus ubagshi y Trochocystoides planus sólo se dispone de un ejemplar incompleto de cada uno. T. planus ha sido puesto recientemente en sinonimia con $S$. ubaghsi (Smith \& Zamora, 2009). Desde el punto de vista de la clasificación supragenérica de los cincta este autor discrepa ampliamente de las propuestas de Friedrich (1993), aspecto que se comentará más adelante.

Gil Cid \& Domínguez (1995a, 1995b) publicaron dos trabajos sobre Cincta de España. En el primero (Gil Cid \& Domínguez, 1995a) describen una nueva especie, Gyrocystis cruzae del Cámbrico medio de Asturias, con material procedente de los fondos del Museo Geominero de Madrid, en cuyas colecciones permanecen clasificados como Decacystis hispanicus. Este material había sido publicado por Hernández-Sampelayo (1935) bajo el nombre de T. bohemicus. Tanto Friedrich (1995) como Zamora \& Álvaro (2010) plantean dudas de que $G$. cruzae sea una especie válida, aunque por el momento se sigue manteniendo como tal. En el siguiente trabajo Gil Cid \& Domínguez (1995b) aportan datos sobre la presencia de Gyrocystis en el Cámbrico medio de Zafra (Badajoz) al volver a estudiar el material de Chauvel \& Meléndez (en Gutiérrez Marco et al. 1984).

Friedrich (1995) describe nuevas formas de cincta de Cerdeña, Marruecos y Gales. Este trabajo también le sirvió para comentar algunas cuestiones referidas a los últimos trabajos aparecidos sobre los cincta españoles (Sdzuy, 1993; Gil Cid \& Domínguez, 1995a, 1995b). Friedrich (1995) se ratifica en su propuesta de clasificación supragenérica de los cincta, y expresa su desacuerdo con las críticas vertidas en Sdzuy (1993). No está de acuerdo en considerar Sucocystidae como sinónimo más reciente de
Trochocystitidae, en lo que coincidimos tras estudiar recientemente la filogenia de los cincta (Smith \& Zamora, 2009). Respecto a los trabajos de Gil Cid \& Domínguez acepta la presencia de Gyrocystis en Zafra (Badajoz), pero plantea que Gyrocystis cruzae pudiera considerarse como un sinónimo posterior de G. platessa.

Gil Cid \& Domínguez (1998) figuran y describen placas aisladas de blastozoo, asignadas a Eocystites y de estilóforo, identificadas como Ceratocystis en el Cámbrico medio de Zafra (Badajoz). Además comentan la presencia de Ceratocystis en Córdoba. Esta es la primera referencia de equinodermos estilóforos en el Cámbrico de España. Domínguez Alonso et al. (2002) sintetizan todos los géneros de carpoideos conocidos en el mundo, incluyendo algunos taxones de cincta definidos en España.

Por último Gil Cid \& Domínguez (2002) publican el eocrinoideo Ubaghsicystis segurae procedente del Cámbrico medio de Los Barrios de Luna. Se trata del representante más antiguo de los eocrinoideos gondwánicos con un pedúnculo compuesto por placas columnares.

Clausen (2004a, 2004b) describe los primeros restos de pelmatozoos del Cámbrico inferior de las Cadenas Ibéricas. Se trata de placas aisladas provenientes del Bilbiliense de Mesones (Zaragoza) y que fueron identificadas como placas basales de la teca de eocrinoideos afines a Rhopalocystis?

Los últimos trabajos sobre equinodermos cámbricos españoles son los publicados por el autor de este trabajo junto a sus colaboradores y se incluyen en la lista bibliográfica de este manuscrito.

\section{Material y técnicas de estudio}

La colección actual de equinodermos cámbricos en la que se está trabajando, incluye a día de hoy, aproximadamente unos 2000 ejemplares completos o parcialmente completos y articulados en la mayoría de los casos. Todo el material proviene de rocas siliciclásticas. El estudio de material silicificado proveniente del ataque mediante ácido de calizas todavía no ha sido abordado. Ejemplos de material español obtenido mediante esta técnica pueden verse en Clausen (2004a, 2004b).

El trabajo de campo ha de llevar un control biostratigráfico muy estricto proporcionado por los trilobites, que son el grupo más utilizado para las divisiones biostratigráficas del Cámbrico (Fig. 6). El muestreo debe ser especialmente intenso en aque- 


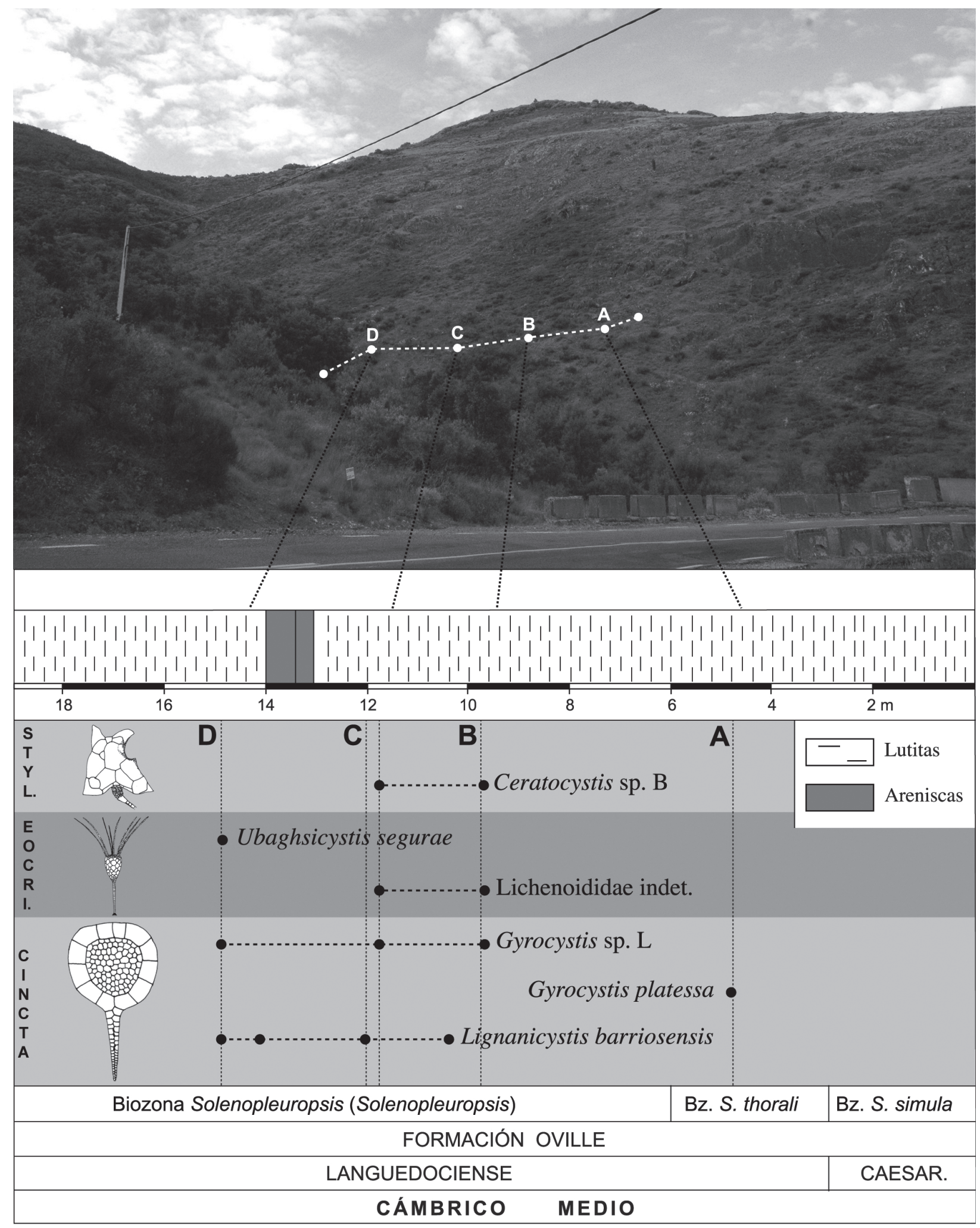

Fig. 6. - Yacimiento cámbrico de Los Barrios de Luna (León) donde se muestra la metodología utilizada para el muestreo de equinodermos en las lutitas basales de la Formación Oville (techo a la izquierda), que se apoyan sobre las calizas de la Formación Láncara (topográficamente por encima). 
llos niveles susceptibles de presentar equinodermos no desarticulados y perfectamente conservados. Las placas aisladas son informativas, pero a nivel taxonómico, sólo permiten una resolución a nivel de clase (Donovan y Paul, 1982). A los niveles con equinodermos completos bien conservados se les conoce en la literatura como «EchinodermLagerstätten» o «starfish beds» (Smith, 1988; Brett et al., 1997; Nebelsick, 2004; Lefebvre, 2007). El trabajo de muestreo ha de ser ingente, ya que muchos grupos de equinodermos son muy minoritarios en las asociaciones fósiles. Por poner algunos ejemplos; existen niveles donde los equinodermos son muy escasos en relación a otros grupos fósiles. Este puede ser el caso de capas del la Formación Oville en Los Barrios de Luna donde la proporción trilobites/equinodermos es aproximadamente 200/1, incluyendo en los trilobites tanto escleritos como ejemplares completos. Existen algunos niveles donde hay gran cantidad de restos de equinodermos, pero es casi imposible encontrar ejemplares articulados susceptibles de ser clasificados (ej. niveles depositados en facies proximales de la base de la Formación Borobia en Purujosa o Torrijo de la Cañada, Cadenas Ibéricas). En estos casos es necesario movilizar varios metros cúbicos de roca para obtener un sólo ejemplar completo. Otros niveles son muy productivos en cuanto a cantidad de ejemplares y estado de conservación; por ejemplo los niveles del techo de la Formación Murero depositados en facies más profundas de Purujosa. En ellos aparecen gran cantidad de ejemplares completos y articulados de diferentes clases de equinodermos representando yacimientos clásicos de EchinodermLagerstätten. En un sólo día de muestreo intenso pueden recuperarse hasta 5-10 ejemplares más o menos completos de cincta y algún gógido (1-2 ejemplares). Otros grupos como los estilóforos, ctenocistoideos, eocrinoideos (no gógidos) y edrioasteroideos son muy raros.

El material se prepara en el laboratorio de manera mecánica mediante percutores eléctricos. En ocasiones los fósiles están totalmente cubiertos por óxidos de hierro que pueden ser eliminados mediante su introducción en disoluciones de ácido oxálico o mediante múltiples réplicas en látex.

Una de las peculiaridades de estos fósiles es que en la mayoría de los casos están conservados como moldes naturales. Para estudiar su morfología detallada se utilizan replicados en látex que son blanqueados con cloruro amónico para ser fotografiados.
Los ejemplares conservados en calcita, que son una minoría, se estudian, o bien disolviendo la calcita en ácido clorhídrico (10\%) para producir moldes artificiales (Fig. 7A), o bien mediante microtomografía computerizada de rayos X (o CT scan) (Rahman \& Zamora, 2009) (Fig. 7C). Existen algunos antecedentes antiguos donde se estudian carpoideos paleozoicos conservados en calcita mediante cortes seriados y peelings de acetato (Jefferies \& Lewis, 1978).

El estudio de moldes naturales mediante escáner CT ha dado recientemente excelentes resultados (Rahman et al., 2010), lo que abre un importante abanico de posibilidades.

El estudio de algunas estructuras replicadas con látex y fotografiadas mediante microscopio electrónico también puede aportar datos de mucho detalle (Zamora et al., 2009a; fig. 7).

De todos los métodos de estudio, la visualización de los ejemplares con lupa binocular y los dibujos a cámara clara siguen representando la principal tarea en el estudio de los equinodermos (Fig. 7D).

El objetivo de todas estas técnicas de observación es el de obtener una reconstrucción lo más fidedigna posible del animal original, incluyendo el mayor número de detalles anatómicos. Toda esta información es básica para incorporar los datos obtenidos en aspectos posteriores como la filogenia o la paleobiología.

\section{Grupos de equinodermos en el Cámbrico Medio de España}

Los equinodermos del Cámbrico de España pueden incluirse en cinco grandes grupos: cincta, eocrinoideos, edrioasteroideos, estilóforos y ctenocistoideos. Estos coinciden con los que propuso Sprinkle (1992) como representantes de la radiación del Cámbrico medio. Sólo existe un clado de equinodermos que todavía no se ha encontrado en el Cámbrico medio de España y que de momento es endémico de Norteamérica, los solutos. Los helicoplacoideos también son endémicos de Norteamérica pero sólo se reconocen en el Cámbrico inferior (Willbur, 2006).

A continuación se presenta una breve descripción de estos grupos con unas indicaciones básicas sobre su anatomía y las cuestiones más relevantes sobre sus hallazgos en nuestro país. Para conocer más aspectos sobre su morfología pueden consultarse Friedrich (1993), Zamora \& Smith (2008) para los 

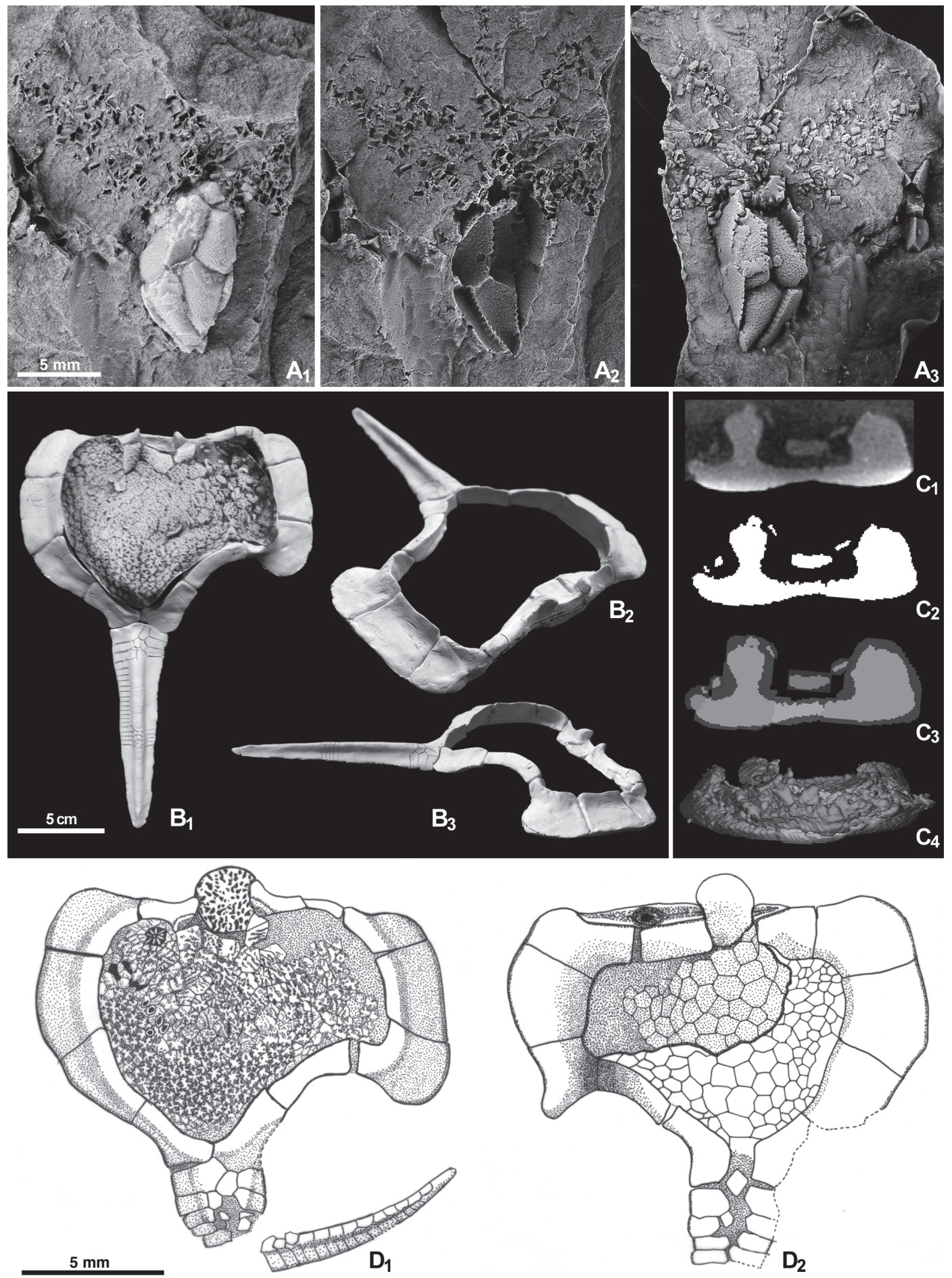

Fig. 7.-Metodologías aplicadas para el estudio de equinodermos cámbricos. A. Proceso de disolución y replicado con látex. $\mathrm{A}_{1}$. Fósil en calcita de un eocrinoideo. $A_{2}$. Molde artificial después de disolver el ejemplar. $A_{3}$. Réplica en látex fotografiada tras su blanqueo con cloruro amónico. B. Modelo a gran escala del cincta Lignanicystis barriosensis Zamora \& Smith utilizado para deducir algunos aspectos sobre su paleobiología. C. Tomografía del cincta Protocinctus mansillaensis Rahman \& Zamora. $\mathrm{C}_{1}$. Sección directamente extraída del microtomógrafo; las diferentes placas del esqueleto aparecen en tonos grises. $\mathrm{C}_{2}$. Sección donde se individualiza el fósil (blanco) de la matriz circundante. $\mathrm{C}_{3}$. Sección con la interpretación de las placas que constituyen el esqueleto en distintas tonalidades $\mathrm{C}_{4}$. Reconstrucción frontal del ejemplar a partir de la combinación de todas las secciones. D. Dibujo a cámara clara del holotipo del cincta Lignanicystis barriosensis Zamora \& Smith en vista dorsal $\left(D_{1}\right)$ y ventral $\left(D_{2}\right)$. 
cincta; Sprinkle (1973) para los eocrinoideos; Smith (1985), Zhao et al. (2010), para los edrioasteroideos; Lefebvre (2003), para los estilóforos y Rahman \& Clausen (2009) para los ctenocistoideos.

\section{Clase Cincta}

Es el grupo más representado en el Cámbrico de España. Son relativamente pequeños y con forma de raqueta (Fig. 8), con un cuerpo plano-convexo (teca) y un apéndice caudal en forma de daga (estela). La cavidad corporal está rodeada por un anillo de grandes placas marginales (cinctus) y se encuentra sellada superior e inferiormente por dos tegumentos de pequeñas placas poligonales dispuestas de manera teselada. La cavidad corporal está perforada por una serie de aberturas (ver discusión en Zamora y Smith, 2008), unas atraviesan el cinctus y otras el tegumento superior de placas (supracentralia). En la parte anterior, dos surcos asimétricos se dirigen hacia una de estas aberturas unánimemente considerada como la boca. Algunos carecen de surco derecho.

En el Cámbrico de España se han identificado especies pertenecientes a dos clados, Gyrocystidae (Fig. 8A, B, C) y Sucocystidae (Fig. 8E, F, G), además de los géneros de cincta primitivos Sotocinctus (Fig. 8D) y Asturicystis. También se encuentra el cincta más antiguo conocido, Protocinctus (Fig. 7C) del Leoniense superior de las Cadenas Ibéricas (Rahman \& Zamora, 2009). Este grupo se extiende desde el Leoniense hasta el Languedociense superior, donde se extinguen.

\section{Clase Eocrinoidea}

Es el segundo grupo en orden de diversidad y abundancia. Posiblemente se trata de un grupo parafilético que comprende varios miembros de los cistoideos (Smith, 1984; Paul, 1988). Las principales innovaciones evolutivas de los eocrinoideos fueron el desarrollo de apéndices exotecales erguidos (braquiolas), para la alimentación, y un órgano de fijación compuesto por numerosas placas globulares, que más tarde dio lugar a tallos formados por placas columnares, y que permitieron a estos organismos elevarse sobre el sustrato para captar alimentos (Sprinkle \& Collins, 2006).

El esqueleto de los eocrinoideos se compone de tres partes principales: braquiolas, teca y tallo (Fig. 9).
Las braquiolas tienen un origen totalmente axial (sensu David et al. 2000), es decir son extensiones de la zona ambulacral, y sirven para la filtración del agua marina y la consiguiente captura de partículas nutritivas. Estas estructuras nacen normalmente de facetas braquiolares, que conectan las braquiolas con los surcos alimenticios y que a su vez convergen en la boca. Existen numerosas diferencias morfológicas entre las braquiolas de los blastozoos y los brazos de los crinoideos, por lo que su posible homología sigue siendo un tema muy discutido (ver resumen en Guensburg \& Sprinkle, 2007; Guensburg et al. 2010). La teca forma el cuerpo principal del organismo y se compone de placas organizadas en ciclos o irregularmente dispuestas. En su interior albergaría la masa visceral. El tallo, que en ocasiones está ausente, permite al organismo elevarse sobre el sustrato. En ocasiones presentan estructuras distales de fijación en forma de disco, maza o ancla (Zamora et al., 2009b).

Existen cuatro grupos de eocrinoideos en el Cámbrico de España.

(i) Los gogidos son el grupo más abundante. Incluye tres especies descritas, una de las cuales pertenece al Cámbrico inferior, Alanisicystis andalusiae (Ubaghs \& Vizcaïno, 1990) (Fig. 9C), y dos al Cámbrico medio; Gogia gondi Ubaghs 1987 (Fig. 9E) y G. parsleyi Zamora 2009 (en Zamora et al., 2009a) (Fig. 9A), además de varios taxones en nomenclatura abierta (Zamora et al., 2009a; Zamora 2010). Sólo se han encontrado gógidos en el Cámbrico inferior de Alanís (Sevilla) y en el Cámbrico medio (Formaciones Murero y Borobia) de las Cadenas Ibéricas.

(ii) Los liquenoıdidos se conocen originariamente de la República Checa (Barrande, 1887; Ubaghs, 1953; Parsley \& Prokop, 2004), y están representados en nuestro país por dos especies de un mismo género (Fig. 9B). Éstas presentan un tallo extremadamente reducido anclado en su parte distal a fragmentos de trilobites. Sus fósiles son muy poco comunes (menos del 5\% en las asociaciones de equinodermos). Se han reconocido miembros de este grupo en la Formación Oville de la Zona Cantábrica y en la Formación Murero de las Cadenas Ibéricas.

(iii) Ubaghsicystis segurae Gil Cid \& Domínguez, 2002 es el único miembro de un nuevo grupo de eocrinoideos (Fig. 9D), que se diferencia de los gogidos en la presencia de un tallo holomérico y por tener un par de brazos que convergen en la zona oral. Sólo se ha encontrado en la Formación Oville 


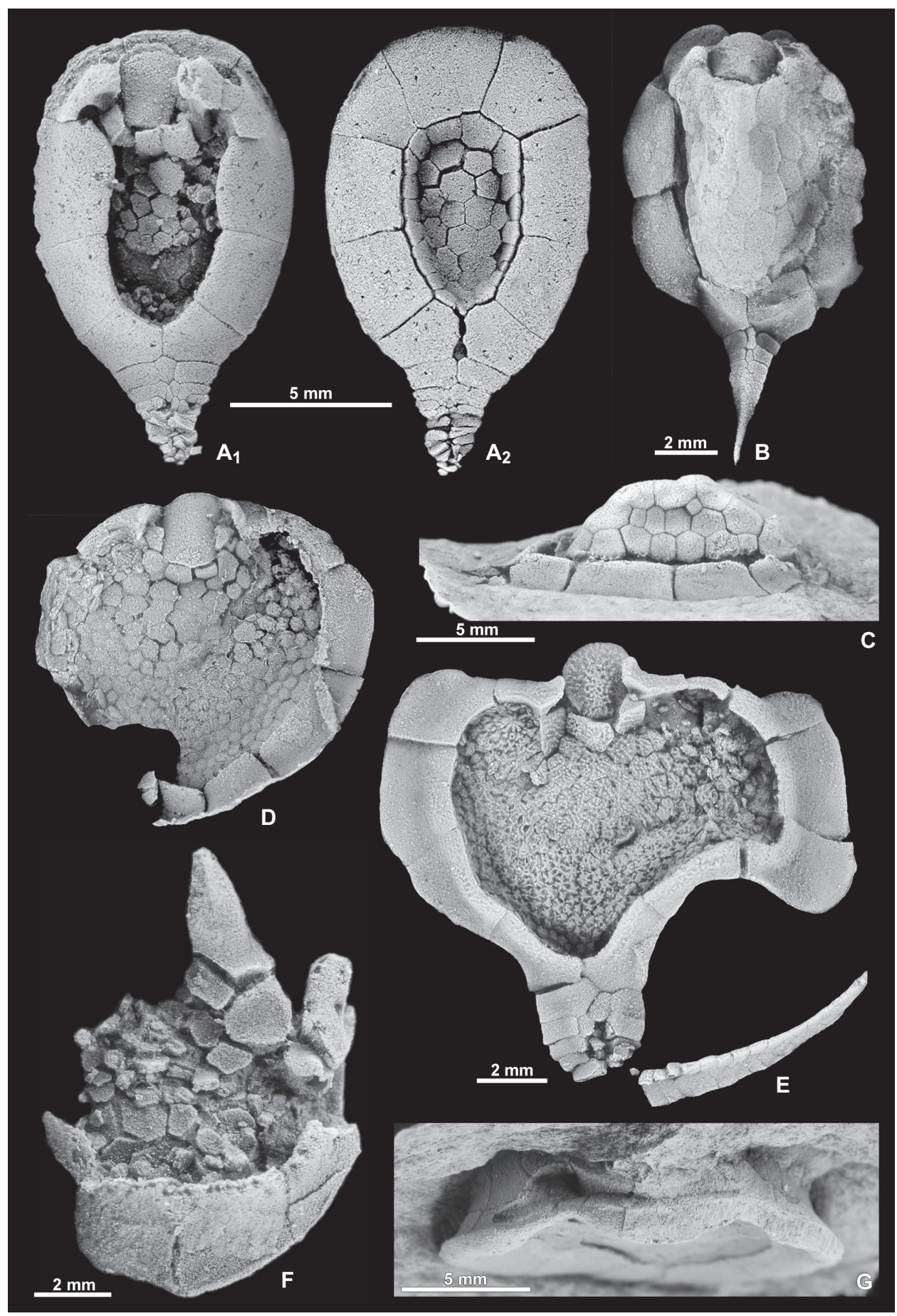

Fig. 8.-Clase Cincta. A. Gyrocystis platessa Jaekel en vista dorsal $\left(A_{1}\right)$ y ventral $\left(A_{2}\right)$ MPZ2004/183, Cámbrico medio de Purujosa (Cadenas Ibéricas). B. Gyrocystis testudiformis Friedrich en vista dorsal, MPZ2009/150, Cámbrico medio de Jarque (Cadenas Ibéricas). C. Gyrocystis testudiformis Friedrich en vista lateral, MPZ2008/4, Cámbrico medio de Purujosa (Cadenas Ibéricas). D. Sotocinctus ubaghsi Sdzuy en vista dorsal, PIW93IX1/1, Cámbrico medio de Soto de Caso (Zona Cantábrica). E. Lignanicystis barriosensis Zamora \& Smith en vista dorsal, MPZ2007/776, Cámbrico medio de los Barrios de Luna (Zona Cantábrica). F. Sucocystis theronensis Cabibel, Termier \& Termier en vista lateral, MPZ2009/138, Cámbrico medio de Torrijo de la Cañada (Cadenas Ibéricas). Oservar la espina central en el lintel, característica de este taxón. G. Undatacinctus melendezi Schroeder en vista frontal, MPZ2009/127, Cámbrico medio de Ateca (Cadenas Ibéricas). Observar la boca y los surcos alimenticios asociados. MPZ: Museo Paleontológico Universidad de Zaragoza (España); PIW: Institut für Paläontologie der Julius Maximilians, Universität Würzburg (Alemania). Todos los ejemplares son réplicas en látex blanqueadas con $\mathrm{NH} 4 \mathrm{Cl}$. 


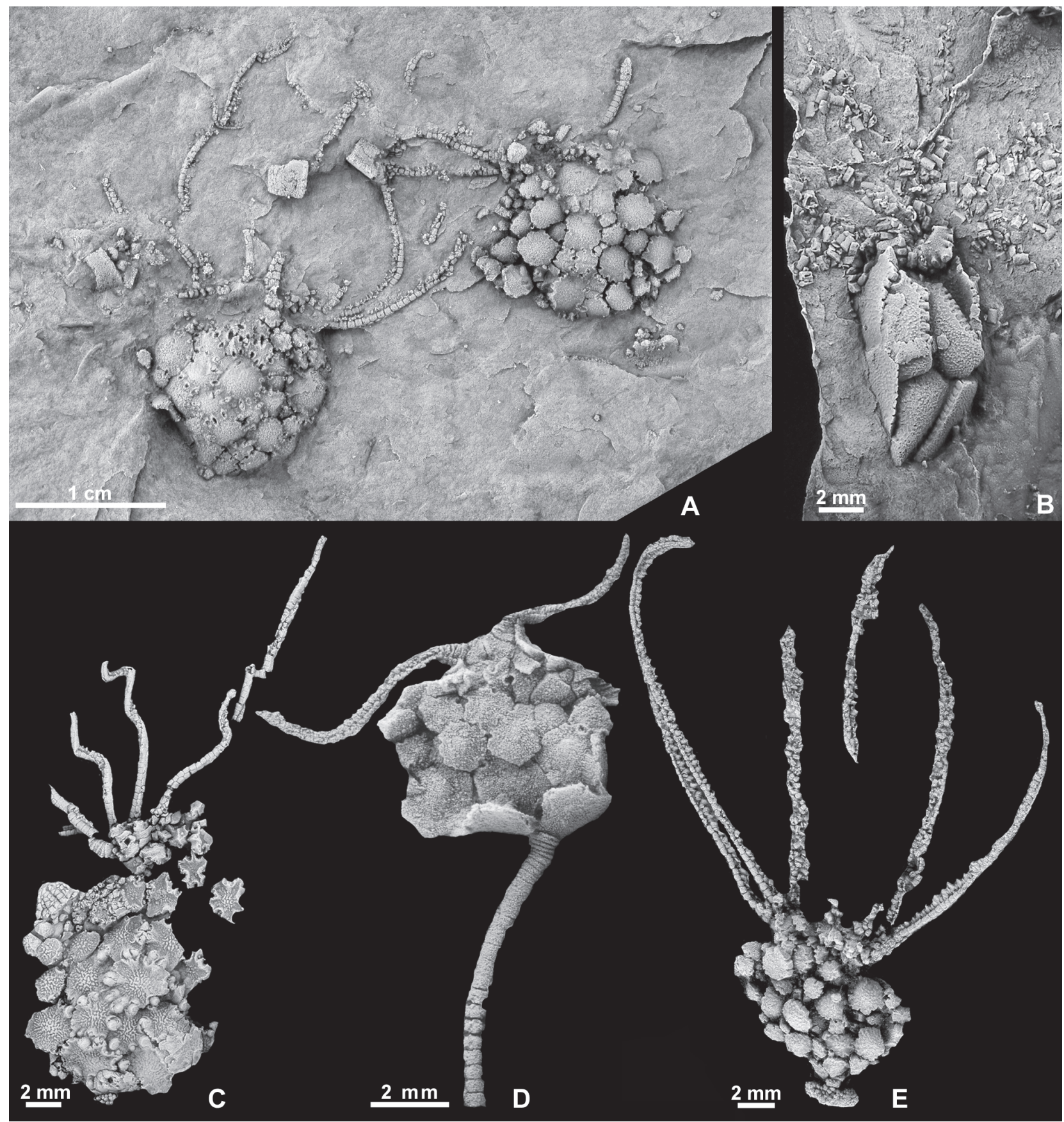

Fig. 9.-Clase Eocrinoidea. A. Dos ejemplares de Gogia parsleyi Zamora, MPZ2004/161, 162, Cámbrico medio de Purujosa (Cadenas Ibéricas). B. Lichenoididae indet., MPZ2009/165, Cámbrico medio de Purujosa (Cadenas Ibéricas). C. Alanisicystis andalusiae Ubaghs \& Vizcaïno, MGM2005/K del Cámbrico inferior de Alanís (Zona de Ossa Morena). D. Ubaghsicystis segurae Gil Cid \& Domínguez, MNCN-I-30849, Cámbrico medio de los Barrios de Luna (Zona Cantábrica). E. Gogia gondi Ubaghs, MPZ2009/164, Cámbrico medio de Murero (Cadenas Ibéricas). MGM: Museo Geominero, Madrid (España); MNCN: Museo Nacional de Ciencias Naturales, Madrid (España). MPZ: Museo Paleontológico Universidad de Zaragoza (España).

Todos los ejemplares son réplicas en látex blanqueadas con $\mathrm{NH} 4 \mathrm{Cl}$. 


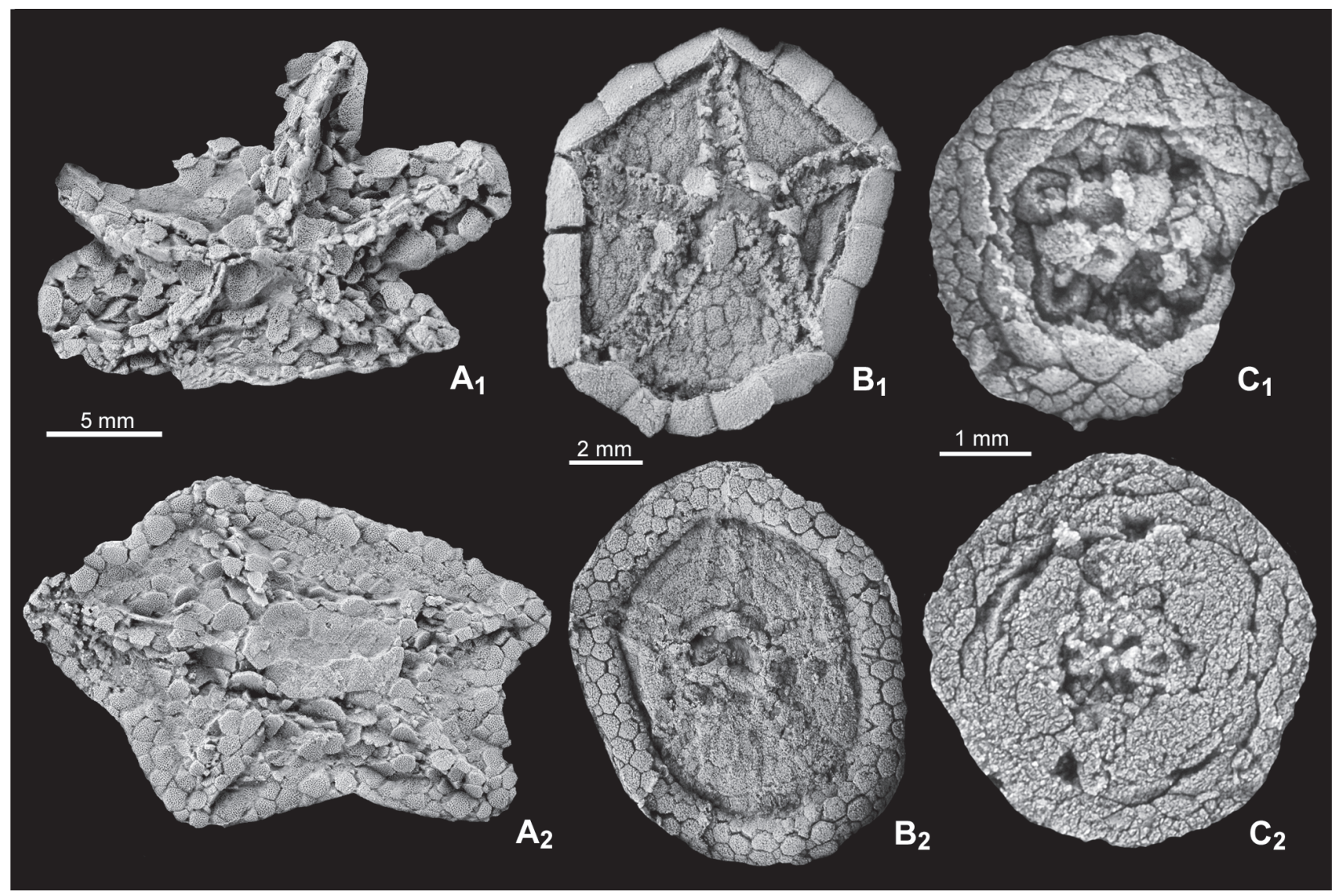

Fig. 10.-Clase Edrioasteroidea. A. Stromatocystitidae indet. en vista oral $\left(A_{1}\right)$ y aboral $\left(A_{2}\right)$. MPZ2007/1890, Cámbrico medio de Murero (Cadenas Ibéricas). B. Cambraster cannati Cabibel, Termier \& Termier, en vista oral $\left(B_{1}\right)$ y aboral $\left(B_{2}\right)$. MPZ2009/1232, Cámbrico medio de Villalengua (Cadenas Ibéricas). C. Protorophus hispanicus Zamora \& Smith, en vista oral $\left(\mathrm{C}_{1}\right)$ y aboral $\left(\mathrm{C}_{2}\right)$. MPZ: Museo Paleontológico Universidad de Zaragoza (España).

Todos los ejemplares son réplicas en látex blanqueadas con $\mathrm{NH} 4 \mathrm{Cl}$.

(Zona Cantábrica), en concreto, en el clásico yacimiento de los Barrios de Luna.

(iv) Los «eocistítidos» constituyen un nuevo grupo de pelmatozoos no descrito formalmente todavía, que en este trabajo se consideran eocrinoideos según su inclusión original (Ubaghs, 1967), aunque seguramente se trata de un grupo diferente. Numerosas localidades cámbricas de Gondwana han proporcionado placas aisladas en forma de estrella, que se conocen con el nombre de Eocystites. Los primeros ejemplares completos y articulados se han encontrado en el Cámbrico de España, en concreto en la Formación Murero de las Cadenas Ibéricas, y permitirán conocer a qué grupo pertenecen. Se trata de equinodermos relativamente grandes (hasta varios centímetros), con una teca compuesta de placas estrelladas, dispuestas de manera teselada y dos apéndices orales formados por series múltiples de placas. Además tienen un tallo dividido en tres partes: una proximal que es pentaradiada, una placa intermedia en forma de cono y una parte distal formada por columnares holoméricos.

\section{Clase Edrioasteroidea}

Son la tercera clase en abundancia y diversidad. Se trata de un grupo de equinodermos cuyo origen se remonta al Cámbrico inferior y cuya historia evolutiva se extiende hasta el Pérmico. Durante este intervalo se conocen más de 60 géneros. La morfología de los edrioasteroideos cámbricos se diferencia ligeramente de sus descendientes, por lo que este apartado está centrado principalmente en la morfología de los primeros.

La teca se divide en dos partes principales (sensu Smith, 1985; ver tambien Zhao et al., 
2010): dorsal (parte inferior de la teca o aboral) y ventral (parte superior de la teca o adoral) que pueden ser de igual o de distinto tamaño dependiendo del género. En la superficie ventral se localizan la mayoría de las estructuras que conectan el interior de la cavidad tecal con el exterior (Fig. $10 \mathrm{~A}_{1}, \mathrm{~B}_{1}, \mathrm{C}_{1}$ ). En ella se localizan los ambulacros, el peristoma, el ano, las epispiras y el hidroporogonoporo. Se diferencian además zonas ambulacrales e interambulacrales. Los ambulacros se sitúan elevados sobre el resto de la teca y presentan un patrón de distribución de tipo 2-1-2 (sensu Sprinkle, 1973), nombrándose con letras de la A a la E. El ano se situaría entre el ambulacro $C$ y el D y el resto se numerarían correlativamente y en sentido horario. Los ambulacros se componen de multitud de placas dispuestas en series. Hay que diferenciar dos grupos de placas principales, placas coberteras (cover plates) y placas ambulacrales basales (flooring plates).

La zona interambulacral está deprimida respecto a los ambulacros y se compone de multitud de placas ensambladas entre sí. En algunas especies, estas placas presentan epispiras en las juntas de unión, excepto en la conexión a las placas ambulacrales, osículos marginales y a las placas del periprocto (Smith, 1985).

Las placas de la zona dorsal (Fig. 10A $2, \mathrm{~B}_{2}, \mathrm{C}_{2}$ ), cuando están calcificadas, pueden dividirse en dos grupos, las que forman el anillo marginal y el resto, situadas en posición central y cerradas por este ani1lo. Las placas de esta superficie, al contrario que en la superficie ventral, nunca presentan epispiras. En el centro se observan en ocasiones varias placas fusionadas, constituyendo un disco de fijación (Fig. 10A 2 ). Una revisión de los edrioasteroideos cámbricos junto a nuevo material descrito de China ha aportado información muy relevante sobre este grupo (Zhao et al., 2010).

Se conocen edrioasteroideos en el Cámbrico español desde hace relativamente poco tiempo (Zamora et al., 2007) y estos pueden incluirse en tres grupos diferentes: estromatocistítidos, cambrastéridos e isorófidos. Los estromatocistítidos (Stromatocystites y formas afines), presentan la zona oral y aboral calcificada, no tienen anillo marginal, y las placas ambulacrales basales son biseriadas mientras que las coberteras se disponen en series de placas múltiples (Fig. 10A). Los cambrastéridos se diferencian de los anteriores en la presencia de un anillo compuesto por placas marginales (Fig. 10B), pero la estructura ambulacral es muy parecida, lo que hace que ambos grupos estén muy relacionados filogenéticamente. Por último los isorófidos tienen placas ambulacrales basales uniseriadas y la zona aboral parcialmente descalcificada. En nuestro país se acaban de publicar los restos más antiguos de este último clado a nivel mundial, representados por Protorophus hispanicus y otro taxón en nomenclatura abierta (Zamora \& Smith, 2010). Los edrioasteroideos presentan un buen registro en las Cadenas Ibéricas donde se encuentran desde el Bilbiliense (Cámbrico inferior) hasta el Languedociense inferior (Cámbrico medio).

\section{Clase Stylophora}

Son el grupo de equinodermos más controvertido que existe desde un punto de vista paleobiológico (ver un resumen en Ruta, 1999). Su esqueleto se compone de dos partes, la teca y el apéndice principal (Fig. 11). Las discrepancias que existen sobre este grupo radican en las diferentes interpretaciones que existen sobre la funcionalidad del apéndice y de las distintas aberturas de la teca. La teca presenta una morfología muy variada, pero a grandes rasgos es asimétrica o con tendencia a la simetría bilateral (Lefebvre, 2003). El apéndice posterior está formado por tres partes, una proximal constituida por series de placas imbricadas, una media formada por el estilocono y otra distal formada por una serie de placas en una disposición uniseriada, que se cierra por series de placas más pequeñas.

En este trabajo se sigue la propuesta de Philip (1979) y Smith (2005), en la que la teca se situaría en posición anterior, donde estaría la boca. Los poros o aberturas situadas a la izquierda de la teca serían considerados como estructuras de salida de una faringe interna. Aún así es necesario clarificar que no todo el mundo sigue esta propuesta y una interpretación alternativa puede consultarse en Lefebvre (2003).

En el Cámbrico de España se han encontrado representantes de dos clados principales pertenecientes al Orden Cornuta: ceratocístidos y coturnocístidos. Los primeros están representados por varias especies pertenecientes al género Ceratocystis, cuyo registro se extiende desde el Caesaraugustiense inferior hasta el Languedociense inferior (Fig. 11A, B). Aunque algunos autores ya habían citado este taxón previamente en nuestro país (Gil Cid \& Domínguez, 1998), el material que ofrecen está desarticulado y sólo puede ser clasificado a 


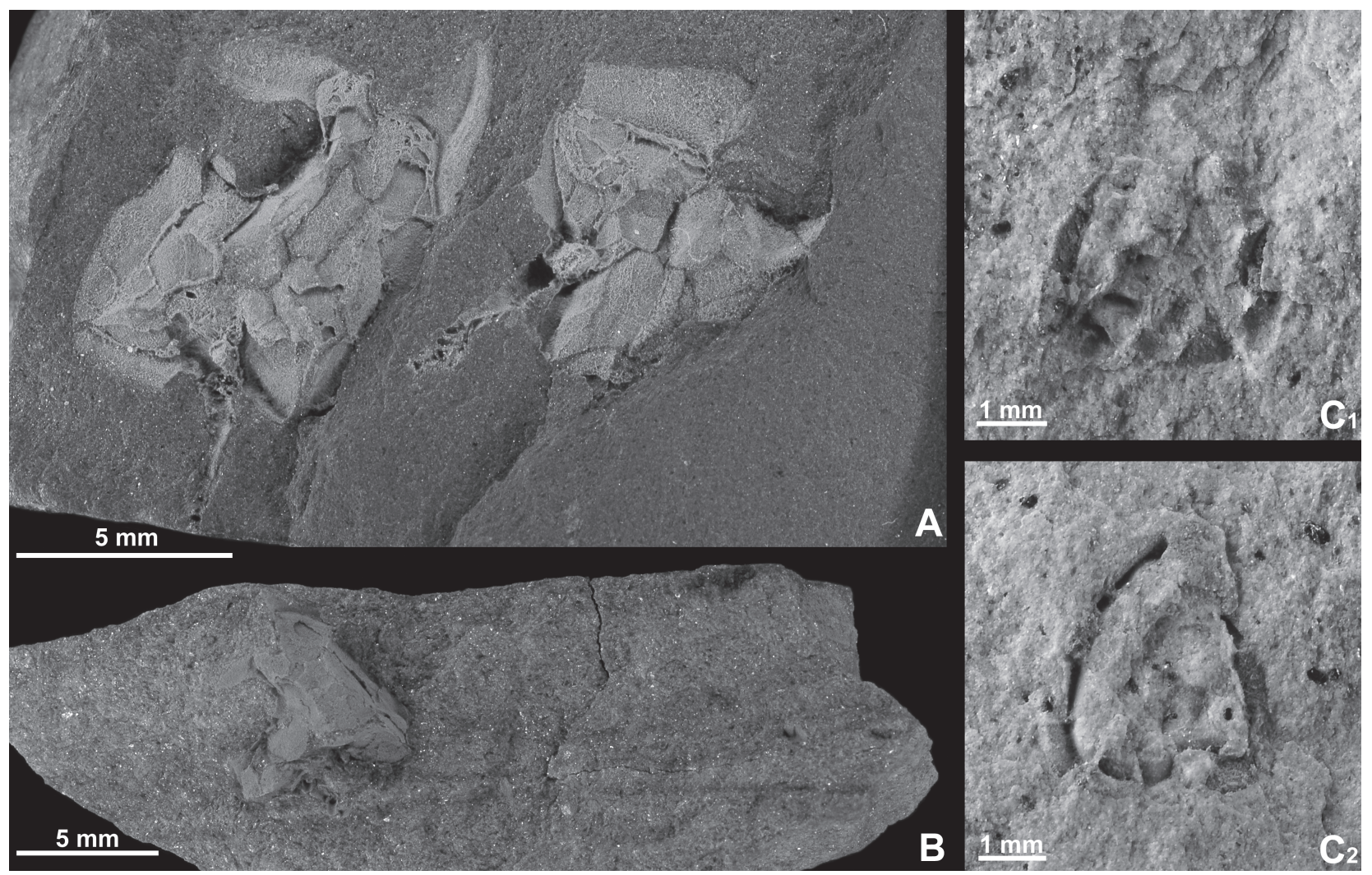

Fig. 11.-Clase Stylophora. A. Dos ejemplares de Ceratocystis sp. A procedentes del Cámbrico medio de Purujosa (Cadenas Ibéricas) (MPZ2009/167-168). B. Ejemplar de Ceratocystis sp. B del Cámbrico medio de los Barrios de Luna (Zona Cantábrica) (MPZ2009/169). C. Coturnocístido del Cámbrico medio de Torrijo de la Cañada (Cadenas Ibéricas) (MPZ2009/170). MPZ: Museo Paleontológico Universidad de Zaragoza (España).

Todos los ejemplares son moldes naturales fotografiados directamente sobre la roca.

nivel de clase. El otro clado representado son los coturnocístidos (Fig. 11C) con dos taxones, uno relacionado con Ponticulocarpus (Sumrall \& Sprinkle, 1999) y otro similar a Cardiocystella (Sumrall et al., 2009). El material proviene del Languedociense inferior.

\section{Clase Ctenocystoidea}

Son un grupo de carpoideos cuyos fósiles se descubrieron relativamente tarde en la historia del estudio de los equinodermos (Robison \& Sprinkle, 1969). Presentan una teca aplanada en sentido dorso-ventral rodeada de placas marginales. Dos tegumentos de pequeñas placas cubren la cavidad corporal. Hasta aquí su plan corporal no es muy diferente al de los cincta. Sin embargo, los ctenocistoideos presentan novedades morfológicas importantes, como la ausencia de apéndice y la presencia de un órgano alimenticio único, el aparato ctenoidal. Esta estructura se conserva especialmente bien en Ctenocystis utahensis Robison \& Sprinkle, 1969 (ver también Rahman \& Clausen, 2009). Se compone de una placa central (supra-oral), de la que irradian hacia izquierda y derecha series de placas ctenoidales. Ventralmente las placas marginales están modificadas en su parte anterior, donde desarrollan una prolongación espinosa que cierran el aparato ctenoidal. Estos organismos presentan una simetría casi bilateral.

Son el grupo peor representado en el Cámbrico de España $(<1 \%$ en las asociaciones de equinodermos) y se conocen a partir de muy pocos ejemplares pertenecientes a tres taxones distintos (Fig. 12). Uno de ellos es muy similar a Etoctenocystis Fatka y Kordule (1985) y los otros dos son seguramente nuevos. Su registro abarca desde el Caesaraugustiense inferior hasta el Languedociense inferior. 


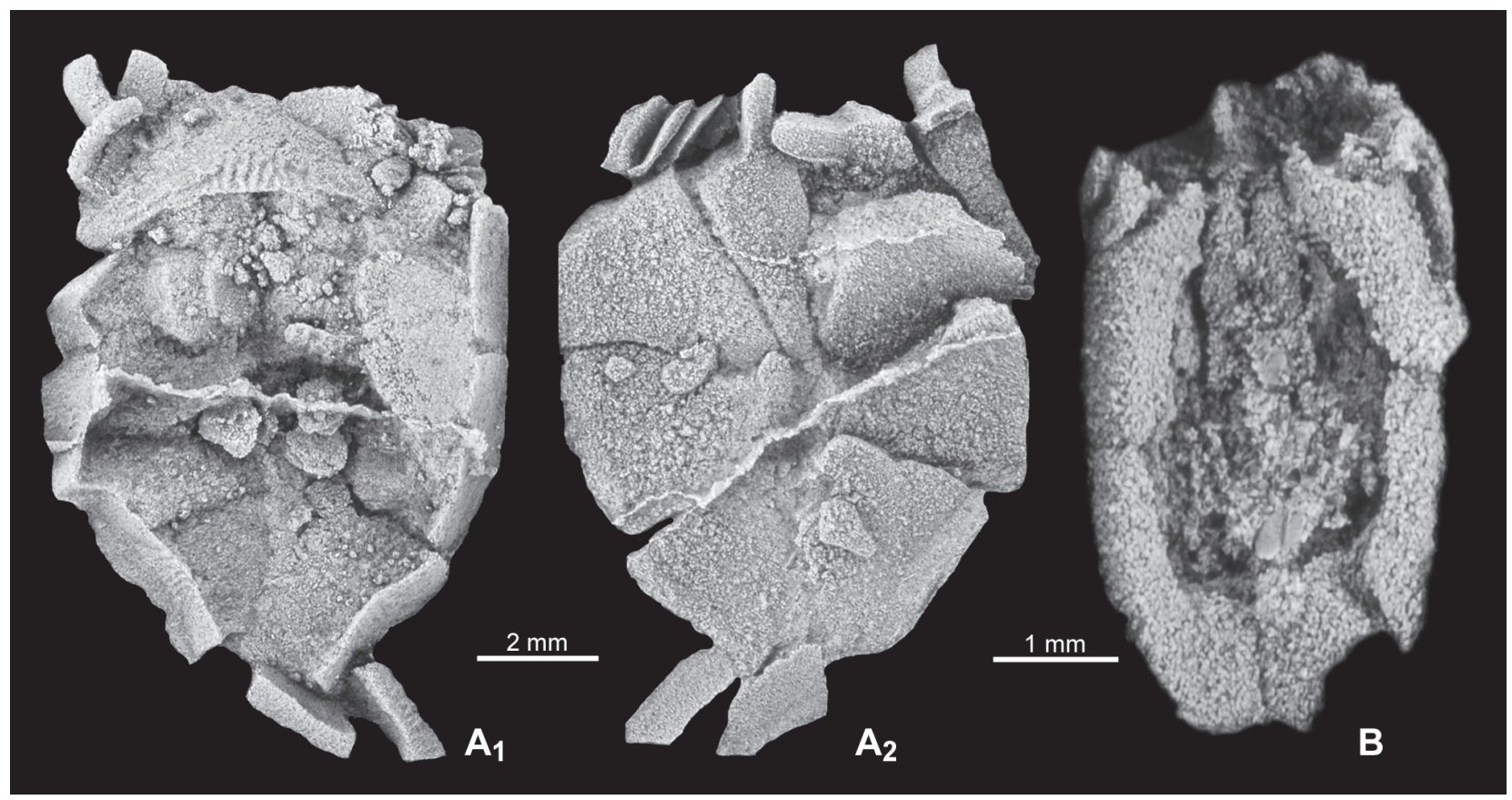

Fig. 12.-Clase Ctenocystoidea. A. Ctenocistoideo (n. gen. n. sp.) en vista dorsal $\left(A_{1}\right)$ y ventral $\left(A_{2}\right)(M P Z 2009 / 1234)$ del Cámbrico medio de Purujosa (Cadenas Ibéricas), donde se observa parte del aparato ctenoidal en la parte anterior. $B$. Etoctenocystis Fatka \& Kordule en vista ventral $\left(A_{2}\right)$ (MPZ2011/42) del Cámbrico medio de Purujosa (Cadenas lbéricas). MPZ: Museo Paleontológico Universidad de Zaragoza (España). Todos los ejemplares son réplicas en látex blanqueadas con $\mathrm{NH} 4 \mathrm{Cl}$..

\section{Consideraciones finales}

A continuación y en base a los datos obtenidos a partir del registro de equinodermos en el Cámbrico español se ofrecen algunas consideraciones finales en tres líneas diferentes: paleobiogeografía, diversidad y origen de equinodermos en el registro fósil. Además, al final de cada uno de los apartados se darán unas indicaciones de hacia dónde deberían de focalizarse los esfuerzos en los próximos años para tratar de resolver los problemas existentes en el actual conocimiento de los equinodermos cámbricos, principalmente en el registro de Gondwana.

\section{Implicaciones paleobiogeográficas}

Hasta el momento sólo existe un trabajo en el que se sintetizan todos los datos sobre la paleobiogeografía de los equinodermos cámbricos de Gondwana (Lefebvre \& Fatka, 2003). Los nuevos hallazgos realizados en España completan considerablemente los datos aportados por estos autores aumentando la distribución paleobiogeográfica de muchos grupos.
Los cincta son de momento endémicos de Gondwana (incluyendo Avalonia) y Siberia. A nivel específico existen taxones con una mayor distribución tanto temporal como espacial. Este es el caso de Gyrocystis platessa encontrado en el Norte de España (Cadenas Ibéricas y Zona Cantábrica) y también en la Montagne Noire (Francia). Algunos taxones que hasta hora se pensaba eran endémicos de la Montagne Noire (Francia), como Sucocystis theronensis o Elliptocinctus barrandei, se han encontrado en nuestro país al muestrear los niveles adecuados (Zamora \& Álvaro, 2010). Taxones clásicos de la República Checa, cómo Trochocystites bohemicus o Trochocystoides parvus continúan restringidos a Bohemia, tras ponerse en sinonimia con Sotocinctus Sdzuy, 1993 un posible Trochocystoides de la Zona Cantábrica (Smith y Zamora, 2009), y tras revisarse todas las citas antiguas de la Península Ibérica (Friedrich, 1993).

Los eocrinoideos muestran coincidencia con otras áreas sólo a nivel de género, con la excepción de la especie Gogia gondi, que también se conoce en Francia. El género Gogia es uno de los que mayor distribución presenta a nivel mundial habiéndose encontrado en Norte América, Méjico, Francia, 
España, Marruecos y seguramente en China (Sprinkle, 1973; Sprinkle \& Collins, 2005; Nardin et al., 2009; Zamora et al., 2009a), pero siempre en paleolatitudes tropicales y subtropicales. La familia lichenoididae, conocida sólo por un género y dos especies de Bohemia (Lichenoides priscus, L. vadosus) (Ubaghs, 1953; Parsley \& Prokop, 2004), se ha reconocido recientemente en el Norte de España. Por último el eocrinoideo Ubaghsicystis segurae permanece endémico de la Zona Cantábrica.

Entre los edrioasteroideos, el género Cambraster se ha descrito en Francia y Australia y recientemente en España (Zamora et al., 2007). En nuestro país se conocen varios estromatocistítidos (Stromatocystites y formas relacionadas), cuyo estudio es muy incipiente como para permitir mayores comparaciones. Otro clado reconocido es el de los edrioasteroideos isorófidos reconocidos por primera vez en el Cámbrico medio ibérico (Zamora y Smith, 2010). Se han descrito dos especies, Protorophus hispanicus Zamora y Smith, 2010 y otra en nomenclatura abierta. Sus fósiles más antiguos hasta el momento procedían del Cámbrico superior de Australia (Smith \& Jell, 1990), aunque empiezan a ser muy abundantes a partir del Ordovícico inferior (Guensburg \& Sprinkle, 1994; Sumrall \& Zamora, 2011).

Estilóforos como Ceratocystis, se reconocen tanto en la Zona Cantábrica como en las Cadenas Ibéricas, y el género se distribuye además por otras regiones gondwánicas como Francia, Bohemia y Alemania (Lefebvre, 2007; Rahman et al., 2010). También aparece en Marruecos aunque todavía no ha sido publicado (Geyer com. pers.). Además se han encontrado algunas placas aisladas asignadas a este género en otras regiones gondwánicas, aunque no se puede asegurar del todo su presencia en base a ellas (Lefebvre, 2007; Rahman et al., 2010).

Aparte de este género, también se conocen en España los únicos miembros de los coturnocístidos conocidos en el Cámbrico medio de Gondwana. Este grupo aparecía hasta el momento en rocas de esta edad sólo en Laurentia (Norteamérica) donde está representado por Cothurnocystis? Ubaghs \& Robison (1988) y Ponticulocarpus Sumrall \& Sprinkle, 1999.

Los ctenocistoideos son un grupo mucho más cosmopolita que los anteriores por lo que su presencia en nuestro país no es extraña. Se han descrito especies que proceden de Gondwana (Polonia, Bohemia, Francia, Australia) y Laurentia (EEUU y Canadá) (Robison \& Sprinkle, 1969; Jell et al., 1985; Ubaghs, 1987; Ubaghs \& Robison, 1988; Dzik
\& Orlowsky, 1995; Fatka \& Kordule, 1985). Si se confirma la presencia del género Etoctenocystis en España implicaría cierta afinidad con los ctenocistoideos de Bohemia. Aun así este grupo necesita ser revisado antes de realizar este tipo de estudios.

La primera conclusión que se deduce de todo esto es que a nivel específico es difícil encontrar faunas comunes con otras regiones (excepto con las más cercanas), lo que marca el gran endemismo de la paleofauna de equinodermos. Es necesario apuntar que el estudio paleobiogeográfico de los taxones de equinodermos del Cámbrico es todavía muy limitado. Existen diversos estudios en los que se aporta material desarticulado de equinodermos tras atacar rocas calizas con ácidos, indicando que los equinodermos estaban presentes en estas zonas (Elicki \& Schneider, 1992; Clausen \& Smith, 2005, 2008; Shinaq \& Elicki, 2007; Clausen et al., 2009), sin embargo, son nulas o muy escasas las referencias en estas mismas regiones de material articulado e identificable. Las futuras investigaciones se deberían centrar en el muestreo sistemático de zonas poco o nada conocidas y la descripción de estas faunas. Por ejemplo, no se conoce ni un sólo equinodermos del Cámbrico inferior y medio de Suramérica; y los taxones de zonas más exploradas como Marruecos están muy poco estudiados.

\section{La diversidad de los equinodermos cámbricos}

Los trabajos sobre la diversidad de equinodermos cámbricos están centrados principalmente en el registro de Norteamérica (Sprinkle, 1992; Sprinkle \& Guensburg, 1997). Aunque se ha realizado algún intento para estimar la diversidad en equinodermos del Cámbrico español (Gil Cid \& Domínguez, 1999), este trabajo carece de una base biostratigráfica adecuada y está basado en muy poco material. Existe un trabajo reciente que muestra la diversidad de los blastozoos durante el Cámbrico y el Ordovícico (Nardin \& Lefebvre, 2010) y sus posibles causas intrínsecas y extrínsecas. En este trabajo se muestra un primer pico de diversidad en el Drumiense (Cámbrico Series 3). Sin embargo este pico tiene ciertos problemas y podría estar exagerado por varias razones: i. La inclusión de los cincta y los ctenocistoideos como blastozoos, interpretación que es muy problemática (ver discusión en Rahman y Zamora, 2009). ii. La correlación del Drumiense en la zona mediterránea de donde proceden un gran número de taxones de equinoder- 
mos. La ausencia en la zona mediterránea de los taxones de trilobites utilizados para la definición del Drumiense (Babcock et al., 2007) dificulta enormemente su correlación con otras áreas. Los nuevos datos quimioestratigráficos (Álvaro et al. 2008) sugieren que muchos taxones previamente considerados de edad Drumiense podrían proceder del Cambico Series 3 piso 5, lo que contrasta con los datos de trilobites ofrecidos por otros autores (Gozalo et al. 2008), iii. Inclusión de taxones muy poco conocidos y cuya inclusión en los blastozoos es muy problemática (ej. Eocystites). El registro tan rico de equinodermos cámbricos que muestra nuestro país, lo hace un lugar idóneo para tratar de establecer los patrones de diversidad en los diferentes clados de este filo y su posterior comparación con otras zonas. Hasta el momento sólo se tienen resultados de los cincta, que son el grupo más abundante y mejor conocido.

En este sentido, Zamora \& Álvaro (2010) revisan todos los hallazgos de cincta en nuestro país, e incluyen también datos de otras regiones como Marruecos y Francia, para tratar de estimar como fue la diversidad en el margen oeste de Gondwana.

El análisis de la diversidad de los cincta muestra su mayor pico durante el Languedociense inferior, aunque esta diversidad se encuentra relacionada en parte por aspectos tafonómicos y en parte por la presencia en el margen oeste de Gondwana de las facies adecuadas durante este intervalo.

Es necesario que las investigaciones futuras traten de interrelacionar la diversidad de todos los grupos de equinodermos. Estos trabajos no deben centrase en meros contajes de taxones por unidad temporal, sino contemplar además otros aspectos que influyen de manera notable en la diversidad observada, como el tipo de facies o la cantidad de afloramientos disponibles.

\section{La «explosión del Cámbrico» y los primeros equinodermos}

La «explosión del Cámbrico» se considera el evento más importante de la historia de la vida, pero sus causas y consecuencias son temas muy controvertidos entre los paleontólogos (Conway Morris, 2006). En unos pocos millones de años numerosos grupos de metazoos aparecieron en los mares, y entre ellos se encontraban los equinodermos.

Los equinodermos presentan un registro fósil bastante completo debido a la facilidad de fosiliza- ción de sus componentes calcíticos. Esto les permite ser unos buenos candidatos para establecer los cambios evolutivos sufridos desde sus primeros representantes. Además, esto los convierte en el principal grupo de deuteróstomos para dicho fin, pudiendo establecer modelos evolutivos que puedan ser comparables a los que se establecen para otros filos animales.

El registro fósil de equinodermos comienza en el Cámbrico inferior con la primera aparición en Norteamérica de los helicoplacoideos y edrioasteroideos (Sprinkle, 1992). A estos les siguen los eocrinoideos (Durham, 1978), que también aparecen a la vez en otras regiones de Gondwana (Ubaghs \& Vizcaïno, 1990; Zhao et al. 2007; Hu et al., 2007). La presencia en el Cámbrico medio de numerosos clados de equinodermos que están ampliamente distribuidos geográficamente (Sprinkle, 1992; Zamora, 2010) sugiere fuertemente que muchos de estos clados debieron originarse durante el Cámbrico inferior, pero sus evidencias fósiles todavía no han sido encontradas.

Sprinkle (1992) mostró que durante el Cámbrico medio se originaron diferentes grupos de equinodermos, sin evidencia alguna de su posible ancestro y sin formas intermedias que permiten unir dichos grupos. Clausen (2004a) demostró a partir de material desarticulado que la diversidad de equinodermos cámbricos en Gondwana es mucho mayor de lo que se pensaba. Esto ha sido corroborado recientemente mediante material articulado procedente de diferentes yacimientos cámbricos españoles (Zamora, 2009). El esfuerzo en los próximos años debe centrarse en el muestreo y estudio de los equinodermos del Cámbrico inferior de Gondwana para tratar de reconstruir los primeros pasos en la evolución de este grupo. Este esfuerzo se podría focalizar de dos maneras; la primera es estimando la diversidad, al menos a nivel de clase, a partir del material desarticulado obtenido de la disolución de calizas. Las calizas de numerosas regiones (i.e. Marruecos, Alemania, España, Cerdeña etc.) proporcionan numerosas placas aisladas de equinodermos (Elicki \& Schneider, 1992; Elicki et al. 2002; Clausen 2004a, b; Elicki 2006; Shinaq \& Elicki, 2007), que deberían ser estudiadas en más detalle para tratar de determinar los grupos de equinodermos presentes en el Cámbrico inferior. La segunda línea de investigación, y quizás la más costosa en tiempo y esfuerzo, es el muestreo de los niveles lutíticos del Cámbrico inferior donde los equinodermos son muy escasos, pero no ausentes. 


\section{AGRADECIMIENTOS}

Los comentarios realizados por dos revisores, Juan Carlos Gutiérrez Marco (Madrid) y M $^{\mathrm{a}}$ Dolores Gil Cid (Madrid), han ayudado a mejorar el presente manuscrito. Imran A. Rahman (Universidad de Manchester) ha revisado la versión inglesa del resumen. Este trabajo es una contribución al Proyecto Consolíder MURERO número CGL2006-12975/BTE del MEC de España-FEDER-EU y al Grupo Consolidado E-17 del Gobierno de Aragón. Las investigaciones han sido financiadas en parte por la Dirección General de Patrimonio Cultural del Gobierno de Aragón.

Agradezco a Isabel Pérez (MEC-FSE) las excelentes fotografías del material y su ayuda en la parte gráfica.

\section{Referencias}

Álvaro, J.J. \& Colchen, M. (2002). Earliest Ordovician pelmatozoan holdfasts from western Europe: the Oryctoconus problem revisited. Eclogae geologicae Helvetiae, 95: 451-459.

Álvaro, J.J.; Bauluz, B.; Pierre, C.; Subías, I. \& Vizcaïno, D. (2008). Carbon chemostratigraphy of the Cambrian-Ordovician transition in a midlatitude mixed platform, Montagne Noire, France. Geological Society of America Bulletin, 120: 962-975. doi:10.1130/ B26243.1

Babcock, L.E.; Robison, R.A.; Rees, M.N.; Peng, S.C. \& Saltzman, M.R. (2007). The Global boundary Stratotype Section and Point (GSSP) of the Drumian Stage (Cambrian) in the Drum Mountains, Utah, USA. Episodes, 30: 84-94.

Barrande, J. (1887). Système Silurien du centre de la Bohème. Vol. VII. Classe des échinodermes, ordre des Cystidées. Praga-Leipzig: 233 pp.

Barrois, C. (1882). Recherches sur les terrains anciens des Asturies et de la Galice. Mémoires de la Société géologique du Nord, 2: 1-630.

Bottjer, D.J.; Davidson, E.H.; Peterson, K.J. \& Cameron, R.A. (2006). Paleogenomics of echinoderms. Science, 314: 956-960. doi:10.1126/science.1132310

Brett, C.E.; Moffat, H.E. \& Taylor, W.L. (1997). Echinoderm taphonomy, taphofacies, and Lagerstätten. Paleontological Society Papers, 3: 147-190.

Colchen, M. \& Ubaghs, G. (1969). Sur des restes d'échinodermes(?) du Cambro-Ordovicien de la Sierra de la Demanda (Burgos-Logroño, Espagne). Bulletin de la Société géologique de France. $7^{\mathrm{éme}}$ ser, 11: 649654.

Conway Morris, S. (2006). Darwin's dilemma: The realities of the Cambrian «explosion». Philosophical Transactions of the Royal Society of London B, 361: 10691083. doi:10.1098/rstb.2006.1846

Clausen, S. (2004a). Les communautés non-récifales à échinodermes et éponges de la transition Cambrien inférieur-moyen dans la région méditerranéenne orientale. $\mathrm{PhD}$ thesis, Université de Lille 1, 341 pp.

Clausen, S. (2004b). New Early Cambrian eocrinoids from the Iberian Chains (NE Spain) and their role in nonreefal benthic communities. Eclogae geologicae Helveticae. 97: 371-379.

Clausen, S. \& Smith, A.B. (2005). Palaeoanatomy and biological affinities of a Cambrian deuterostome (Stylophora): Nature, 438: 351-354. doi:10.1038/nature04109

Clausen, S. \& Smith, A.B. (2008). Stem structure and evolution in the earliest pelmatozoan echinoderms: Journal of Paleontology, 82: 737-748. doi:10.1666/ 06067.1

Clausen, S.; Jell, P.A.; Legrain.; X. \& Smith A.B. (2009). Pelmatozoan arms from the Middle Cambrian of Australia: bridging the gap between brachioles and brachials?: Lethaia, 42: 283-296. doi:10.1111/j.15023931.2008.00145.x

David, B, Lefebvre, B, Mooi, R, Parsley, RL. (2000). Are homalozoans echinoderms? An answer from the extraxial-axial theory. Paleobiology, 26, 529-555. doi:10.1666/0094-8373(2000)026<0529:AHEA$\mathrm{AF}>2.0 . \mathrm{CO} ; 2$

Domínguez Alonso, P.; Jefferies, R.P.S. \& Gil Cid, D. (2002). An annotated check-list on genera and species of carpoids. Coloquios de Paleontología, 53, 33-68.

Donovan, S.K. \& Paul, C.R.C. (1982). Lower Cambrian echinoderm plates from Comley, Shropshire, England. Geological Magazine, 119: 611-614. doi:10.1017/S0016756800027102

Durham, J.W. (1974). Systematic position of Eldonia ludwigi Walcott, Journal of Paleontology, 48: 750755.

Durham, J.W. (1978). A lower Cambrian eocrinoid. Journal of Paleontology, 52: 195-199.

Dzik, J. \& Orlowski, S. (1995). Primitive ctenocystoid echinoderm from the earliest Middle Cambrian of Poland. Annales de Paléontologie, 81: 17-35.

Elicki, O. (2006), Microbiofacies analysis of Cambrian offshore carbonates from Sardinia (Italy): environment, reconstruction and development of a drowning carbonate platform, Carnets de Géologie/Notebooks on Geology, Brest, 1: 1-22.

Elicki, O \& Schneider, J. (1992). Lower Cambrian (Atdabanian/Botomian) Shallow-Marine Carbonates of the Görlitz Synclinorium (Saxoniy/Germany). Facies, 26: 55-66. doi:10.1007/BF02539793

Elicki, O.; Schneider J.W. \& Shinaq, R. (2002). Prominent facies from the Lower/Middle Cambrian of the Dead Sea area (Jordan) and their palaeodepositional significance. Bulletin de la Société géologique de France, 173: 147-152. doi:10.2113/173.6.547

Fatka, O. \& Kordule, V. (1985). Etoctenocystis bohemica gen. et sp. nov., new ctenocystoid from Czechoslovakia (Echinodermata, middle Cambrian). Vestník Ústredního ústavu geologického, 60: 225-230.

Friedrich, W.P. (1993). Systematik und Funktionsmorphologie mittelkambrischer Cincta (Carpoidea, Echinodermata). Beringeria, 7: 190 pp.

Friedrich, W.P. (1995). Neue Nachweise mittelkambrischer Cincta (Carpoidea, Echinodermata) aus Marokko, Sardinien und Süd-Wales. Beringeria, Special Issue, 2: 255-269. 
Gil Cid, M.D. \& Domínguez Alonso, P. (1995a). Gyrocystis cruzae, una nueva especie de Cincta (Echinodermata Carpoidea) del Cámbrico Medio del Ferredal de Quintana (Asturias, España). Boletín Geológico y Minero, 106: 517-531.

Gil Cid, M.D. \& Domínguez Alonso, P. (1995b). Presencia de Gyrocystis Jaekel,1918 en el Cámbrico Medio de Zafra (Badajoz). Revista de la Sociedad Geológica de España, 8: 99-110.

Gil Cid, M.D. \& Domínguez Alonso, P. (1998). «Carpoidea» and Pelmatozoa from the Middle Cambrian of Zafra (SW Spain). In: Echinoderms (Mooi R. \& Telford M., eds.). A.A. Balkema, Rotterdam, 93-98.

Gil Cid, M.D. \& Domínguez Alonso, P. (1999). Diversidad de equinodermos y carpoideos en el Cámbrico Inferior y Medio de la Península Ibérica. Coloquios de Paleontología, 50: 105-116.

Gil Cid, M.D. \& Domínguez Alonso, P. (2002). Ubaghsicystis segurae nov. gen. y sp., nuevo Eocrinoide (Echinodermata) del Cámbrico Medio del Norte de España. Coloquios de Paleontología, 53: 21-32.

Gislén, T. (1927). A new Spanish carpoid. Arkiv för Zoo$\log i, 19 \mathrm{~B}: 1-3$.

Gozalo, R.; Liñán, E.; Gámez-Vintaned, J.A.; Dies, M.E.; Chirivella, J.B.; Zamora, S.; Esteve, J. \& Mayoral, E. (2008). The Cambrian of the Cadenas Ibéricas (NE Spain) and its Trilobites. In: Rábano, I.,Gozalo, R. \& García-Bellido, D. (eds.), Advances in trilobite research. Cuadernos del Museo Geominero, 9. Instituto Geológico y Minero de España, Madrid: 137-151.

Guensburg, T.E. \& Sprinkle, J. (1994). Revised phylogeny and functional interpretation of the Edrioasteroidea based on new taxa from the Early and Middle Ordovician of western Utah. Fieldiana (Geology), 29: $1-43$.

Guensburg, T.E. \& Sprinkle, J. (2007). Phylogenetic implications of the Protocrinoida: Blastozoans are not ancestral to crinoids. Annales de Paléontologie, 93: 277-290. doi:10.1016/j.annpal.2007.09.005

Guensburg, T.E.; Mooi, R.; Sprinkle, J.; David, B. \& Lefebvre, B. (2010). Pelmatozoan arms from the midCambrian of Australia: bridging the gap between brachioles and brachials? Comment: there is no bridge. Lethaia, 43: 432-440.

Gutiérrez-Marco, J.C.; Chauvel, J.; Meléndez, B. \& Smith, A.B. (1984). Los equinodermos (Cystoidea, Homalozoa, Stelleroidea, Crinoidea) del Paleozoico inferior de los Montes de Toledo y Sierra Morena (España). Estudios Geológicos, 40: 421-253. doi:10.3989/egeol.84405-6680

Hernández-Sampelayo, P. (1935). El Sistema Cambriano. Explicación del Nuevo Mapa Geológico de España en Escala 1:1.000.000, I. El Sistema Cambriano. Memorias del Instituto Geológico y Minero de España, 41, 291-528.

Hu, S.-X.; Luo, H.-L.; Hou, S.-G. \& Erdtmann, B.D. (2007). Eocrinoid echinoderms from the Lower Cambrian Guanshan fauna in Wuding, Yunnan, China. Chinese Science Bulletin, 52: 717-719. doi:10.1007/ s11434-007-0083-6
Jefferies, R.P.S. \& Lewis, D.N. (1978). The English Silurian fossil Placocystites forbesianus and the ancestry of the vertebrates. Philosophical Transactions of the Royal Society (B), 282: 205-323. doi:10.1098/ rstb.1978.0013

Jell, P.A.; Burrett, C.F. \& Banks, M.R. (1985): Cambrian and Ordovician echinoderms from eastern Australia. Alcheringa, 9: 183-208. doi:10.1080/03115518508618967

Josopait, V. \& Schmitz, U. (1971): Beitrag zur Stratigraphie im Unter-und Mittel-Kambrium der Sierra de la Demanda (NE-Spanien), Münstersche Forschungen zur Geologie und Paläontologie, 19: 85-99.

Lefebvre, B. (2003). Functional Morphology of Stylophoran Echinoderms. Palaeontology, 46: 511-555. doi:10.1111/1475-4983.00309

Lefebvre, B. (2007). Early Palaeozoic palaeobiogeography and palaeoecology of stylophoran echinoderms. Palaeogeography, Palaeoclimatology, Palaeoecology, 245: 156-199. doi:10.1016/j.palaeo.2006.02.021

Lefebvre, B. \& Fatka, O. (2003). Palaeogeographical and palaeoecological aspects of the Cambrian-Ordovician radiation of echinoderms in Gondwanan Africa and peri-Gondwanan Europe. Palaeogeography, Palaeoclimatology, Palaeoecology, 195: 73-97. doi:10.1016/S0031-0182(03)00303-1

Le Menn, J. \& Meléndez, B. (1988). Nouvelle interpretation des Echinodermes de Collado de Tosas (Gerona, Espagne). Estudios Geológicos, 44, 473-476. doi:10.3989/egeol.884456563

Liñán, E.; Gonçalves, F.; Gámez Vintaned, J.A. \& Gozalo, R. (1997). Evolución paleogeográfica del Cámbrico de la Zona de Ossa-Morena basada en el registro fósil. In: Estudios sobre a Geologia da Zona de Ossa-Morena (Maciço Ibérico). Livro de Homenagem ao Prof. Francisco Gonçalves (Alexandre Araújo A. \& Pereira M.F. eds.), 1-26.

Liñán, E.; Gozalo, R.; Palacios, T.; Gámez Vintaned, J.A.; Ugidos, J.M. \& Mayoral, E. (2002): Cambrian. In: The Geology of Spain (Gibbons W. \& Moreno T., eds.). The Geological Society, London, 17-29.

Lotze, F. (1961). Das Kambrium Spaniens. Teil I: Stratigraphie. Akademie der Wissenschaften und der Literatur, Abhandlungen der mathematisch-naturwissenschaftlichen Klasse, 1961 (6): 283-498.

Mallada, L. (1875). Sinopsis de las especies fósiles que se han encontrado en España. Sistema Siluriano. Boletín de la Comisión del Mapa Geológico de España, 2: 1-161.

Mallada, L. \& Buitrago, J. (1878). La fauna primordial a uno y otro lado de la Cordillera Cantábrica. Boletín de la Comisión del Mapa Geológico de España, 5: 177-194.

Meléndez, B. (1952a). Consideraciones de: Gislen, T., Un Carpoideo Nuevo de España. Publicaciones extranjeras sobre Geología de España, 6: 190-191.

Meléndez, B. (1952b). Los Carpoideos de España. Las Ciencias, 17: 497-516.

Meléndez, B. (1954). Los Trochocystites del Pirineo. Boletín de la Real Sociedad Española de Historia Natural, 51, 97-105.

Monreal, L.N. (1879). Datos geológicos a cerca de la provincia de León, recogidos durante la campaña 1878 
a 1879. Boletín de la Comisión del Mapa Geológico de España, 6: 311-320.

Nardin, E.; Almazan Vázquez, E. \& Buitrón Sánchez, B.E. (2009). First report of Gogia (Eocrinoidea, Echinodermata) from the Early-Middle Cambrian of Sonora (Mexico), with biostratigraphical and palaeoecological comments. Geobios, 42: 233-242. doi:10.1016/ j.geobios.2008.10.003

Nardin, E. \& Lefebvre, B. (2010). Unravelling extrinsic and intrinsic factors of the Early Palaeozoic diversification of blastozoan Echinoderms, Palaeogeography, Palaeoclimatology, Palaeoecology, 294: 142-160. doi:10.1016/j.palaeo.2010.01.005

Nebelsick, J.H. (2004). Taphonomy of Echinoderms: A review. In: Echinoderms Munich. Proceedings of the 11th International Echinoderm Meeting (Heinzeller Th. \& Nebelsick J.H. eds.). Taylor and Francis, Rotterdam: 471-478.

Parsley, R.L. \& Prokop, R.J. (2004). Functional morphology and palaeoecology of some sessile Middle Cambrian echinoderms from Barrandian region of Bohemia, Bulletin of Geosciences, 79: 147-156.

Paul, C.R.C. (1988): The phylogeny of the cystoids. En: Echinoderm Phylogeny and Evolutionary Biology (Paul, C.R.C. \& Smith, A.B., eds.). Clarendon Press. Oxford, 199-213.

Philip, G.M. (1979). Carpoids- echinoderms or chordates? Biological Reviews, 54: 439-471. doi:10.1111/ j.1469-185X.1979.tb00845.X

Prado, C.; Verneuil, E. de \& Barrande, J. (1860). Sur l'existence de la faune primordiale dans la chaîne cantabrique. Bulletin de la Société géologique de France, $2^{\text {éme }}$ ser., 17, 516-554.

Quesada, C. (1991). Geological constraints on the Paleozoic tectonic evolution of tectonostratigraphic terranes in the Iberian Massif. Tectonophysics, 185, 225-245. doi:10.1016/0040-1951(91)90446-Y

Rahman, I.A. \& Clausen, S. (2009). Re-evaluating the palaeobiology and affinities of the Ctenocystoidea (Echinodermata). Journal of Systematic Palaeontology, 7, 413-426. doi:10.1017/S1477201909990046

Rahman I.A. \& Zamora S. (2009). The oldest cinctan carpoid (stem-group Echinodermata) and the evolution of the water vascular system. Zoological Journal of the Linnean Society, 157: 420-432. doi:10.1111/j.10963642.2008.00517.x

Rahman, I.A.; Zamora, S. \& Geyer, G. (2010). The oldest stylophoran echinoderm: a new Ceratocystis from the Middle Cambrian of Germany. Palaeontologische Zeitschrift, 84: 227-237. doi:10.1007/s12542009-0039-z

Robison, R.A. \& Sprinkle, J. (1969). Ctenocystoidea: new class of primitive echinoderms. Science. 166, 1512-1514. doi:10.1126/science.166.3912.1512

Ruta, M. (1999). A brief review of the stylophoran debate. Evolution and Development, 1: 123-135. doi:10.1046/j.1525-142x.1999.99008.x

Schröeder, R. (1972). Nota preliminar sobre los Carpoideos del Cámbrico Medio de Asturias y León. Breviora Geológica Astúrica, 2: 17-22.
Schröeder, R. (1973). Carpoideen aus dem Mittelkambrium Nordspaniens. Palaeontographica Abteilung A. 141: 119-142.

Sdzuy, K. (1993). Early Cincta (Carpoidea) from the Middle Cambrian of Spain. Beringeria. 8: 189-207.

Shinaq, R. \& Elicki, O. (2007). The Cambrian sedimentary succession from the Wadi Zerqa Ma'in (northeastern Dead Sea area, Jordan): lithology and fossil content. Neues Jahrbuch für Geologie und Paläontologie, 243: 255-271. doi:10.1127/00777749/2007/0243-0255

Smith, A.B. (1985). Cambrian eleutherozoan echinoderms and the early diversification of edrioasteroids. Palaeontology, 28: 715-756.

Smith, A.B. (1988). Patterns of diversification and extinction in Early Palaeozoic echinoderms. Palaeontology, 31: 799-828.

Smith, A.B. (2005). The pre-radial history of echinoderms. Geological Journal, 40: 255-280. doi:10.1002/ gj. 1018

Smith, A.B. \& Jell, P.A. (1990). Cambrian edrioasteroids from Australia and the origins of starfishes. Memoirs of the Queensland Museum. 28: 715-778.

Smith, A.B. \& Zamora, S. (2009). Rooting phylogenies of problematic fossil taxa; a case study using cinctans (stem-group echinoderms). Palaeontology, 52: 803822. doi:10.1111/j.1475-4983.2009.00880.x

Sprinkle, J. (1973). Morphology and Evolution of Blastozoan Echinoderms. Harvard University Museum of Comparative Zoology, Special Publication, 283 pp.

Sprinkle, J. (1976). Biostratigraphy and paleoecology of Cambrian echinoderms from the Rocky Mountains. Brigham Young University Geology Studies, 23: 61-74.

Sprinkle, J. (1992). Chapter 11. Radiation of Echinodermata. In: Origin and early evolution of the metazoa (Lipps J.H. \& Signor P.W. eds.). Plenum Press, New York, 375-398.

Sprinkle, J. \& Collins, D. (2006). New eocrinoids from the Burgess Shale, southern British Columbia, Canada, and the Spence Shale, northern Utah, USA. Canadian Journal of Earth Sciences, 43: 303-322. doi:10.1139/E05-107

Sprinkle, J. \& Guensburg, T.E. (1997). Early radiation of echinoderms. In: Geobiology of Echinoderms (Waters, J.A. y Maples, C.L., eds.). Palaeontological Society papers, 3, 205-224.

Sumrall, C.D. \& Sprinkle, J. (1999). Ponticulocarpus, a new cornute-grade stylophoran from the Middle Cambrian Spence Shale of Utah. Journal of Paleontology, 73: 886-891.

Sumrall C.D. \& Zamora S. (2011). Ordovician Edrioasteroids from Morocco: Faunal exchanges across the Rheic Ocean. Journal of Systematic Palaeontology, in press.

Sumrall, C.D.; Sprinkle, J. Pruss, S. \& Finnegan, S. (2009). Cardiocystella, a new cornute stylophoran from the Upper Cambrian Whipple Cave Formation, eastern Nevada, USA. Journal of Paleontology, 83: 307-312. doi:10.1666/08-114.1

Ubaghs, G. (1953). Notes sur Lichenoides priscus Barrande, Eocrinoïde du Cambrien moyen de la Tché- 
coslovaquie. Institut Royal des Sciences Naturelles de Belgique, 29: 1-24.

Ubaghs, G. (1967). Eocrinoidea. In Treatise on Invertebrate Paleontology, Pt. S, Echinodermata 1(2), (Moore, R.C., ed.). New York, Geological Society of America (and Univ. Kansas Press): S455-495.

Ubaghs, G. (1987). Echinodermes nouveaux du Cambrien moyen de la Montagne Noire (France). Annales de Paléontologie, 73: 1-27.

Ubaghs, G. \& Robison, R.A. (1988). Homalozoan echinoderms of the Wheeler Formation (Middle Cambrian) of western Utah. The University of Kansas Paleontological Contributions, 120: 1-17.

Ubaghs, G. \& Vizcaïno, D. (1990). A new eocrinoid from the Lower Cambrian of Spain. Palaeontology, 33: 259-256.

Wilbur, B.C. (2006). Reduction in the number of Early Cambrian helicoplacoid species. Palaeoworld, 15, 283-293.

Zamora, S. (2009). Equinodemos del Cámbrico medio de las Cadenas Ibéricas y de la Zona Cantábrica (Norte de España). Tesis Doctoral, Universidad de Zaragoza: 307 pp.

Zamora, S. (2010). Middle Cambrian echinoderms from North Spain show echinoderms diversified earlier in Gondwana. Geology, 38, 507-510.

Zamora, S.; Liñán, E.; Domínguez Alonso, P.; Gozalo, R. \& Gámez Vintaned, J.A. (2007). A Middle Cambrian edrioasteroid from the Murero biota (NE Spain) with Australian affinities. Annales de Paleontologie, 93: 249-260.

Zamora, S. \& Smith, A.B. (2008). A new Middle Cambrian stem-group echinoderm from Spain: Palaeobiological implications of a highly asymmetric cinctan. Acta Palaeontologica Polonica, 53: 207-220.
Zamora, S.; Gozalo, R. \& Liñán, E. (2009a). Middle Cambrian gogiid echinoderms from Northeast Spain: Taxonomy, palaeoecology and palaeogeographic implications. Acta Paleontologica Polonica, 54: 253265.

Zamora, S.; Álvaro, J.J. \& Vizcaïno, D. (2009b). Pelmatozoan echinoderms from the Cambrian-Ordovician transition of the Iberian Chains (NE Spain): early diversification of anchoring strategies. Swiss Journal of Geosciences, 102: 43-55.

Zamora, S. \& Álvaro, J.J. (2010). Testing for a decline in diversity prior to extintion: Languedocian (latest midCambrian) distribution of cinctans (Echinodermata) in the Iberian Chains, NE Spain. Palaeontology, 53: 1349-1368.

Zamora, S. \& Smith, A.B. (2010). The oldest isorophid edrioasteroid (Echinodermata) and the evolution of attachment strategies in Cambrian edrioasteroids. Acta Palaeontologica Polonica, 55: 487-494.

Zamora, S.; Clausen, S.; Álvaro, J.J. \& Smith, A.B. (2010). Pelmatozoan echinoderms as colonizers of carbonate firmgrounds in mid-Cambrian high energy environments. Palaios, 25, 764-768.

Zhao, Y-L.; Parsley, R.L. \& Peng, J. (2007). Early Cambrian echinoderms from Guizhou Province, South China. Palaeogeography, Palaeoclimatology, Palaeoecology, 254: 317-327.

Zhao, Y.; Sumrall, C.D.; Parsley, R L. \& Peng, J. (2010): Kailidiscus, a new plesiomorphic edrioasteroid from the basal middle Cambrian Kaili Biota of Guizhou Province, China. Journal of Paleontology, 84: 668-680.

Recibido el 3 de marzo de 2010

Aceptado el 4 de noviembre de 2010

Publicado online el 10 de mayo de 2011 